\title{
Unravelling the Turing bifurcation using spatially varying diffusion coefficients
}

\author{
Debbie L. Benson ${ }^{1,2}$, Philip K. Maini ${ }^{1, \star}$, Jonathan A. Sherratt ${ }^{3}$ \\ ${ }^{1}$ Centre for Mathematical Biology, Mathematical Institute, 24-29 St Giles', Oxford \\ OX13LB, UK. e-mail: maini@maths.ox.ac.uk \\ ${ }^{2}$ Magdalen College, Oxford, OX1 4AU, UK \\ ${ }^{3}$ Department of Mathematics, Heriot-Watt University, Edinburgh EH14 4AS, UK. \\ e-mail: J.A. Sherratt@ma.hw.ac.uk
}

Received: 10 January 1996/Revised version: 3 July 1996

\begin{abstract}
The Turing bifurcation is the basic bifurcation generating spatial pattern, and lies at the heart of almost all mathematical models for patterning in biology and chemistry. In this paper the authors determine the structure of this bifurcation for two coupled reaction diffusion equations on a two-dimensional square spatial domain when the diffusion coefficients have a small explicit variation in space across the domain. In the case of homogeneous diffusivities, the Turing bifurcation is highly degenerate. Using a two variable perturbation method, the authors show that the small explicit spatial inhomogeneity splits the bifurcation into two separate primary and two separate secondary bifurcations, with all solution branches distinct. This splitting of the bifurcation is more effective than that given by making the domain slightly rectangular, and shows clearly the structure of the Turing bifurcation and the way in which the various solution branches collapse together as the spatial variation is reduced. The authors determine the stability of the solution branches, which indicates that several new phenomena are introduced by the spatial variation, including stable subcritical striped patterns, and the possibility that stable stripes lose stability supercritically to give stable spotted patterns.
\end{abstract}

Key words: Turing bifurcation - Reaction diffusion - Weakly nonlinear - Pattern formation

\footnotetext{
^ Author for correspondence
} 


\section{Introduction}

The Turing bifurcation (Turing, 1952) is the basic bifurcation generating spatial pattern, and lies at the heart of almost all mathematical models for patterning in embryology, ecology and elsewhere in biology and chemistry (see Murray, 1989, for review). The key idea underlying the Turing mechanism is that a homogeneous equilibrium can be stable to homogeneous perturbations, but unstable to certain spatially varying perturbations, leading to a spatially varying steady state, that is, a spatial pattern. The simplest system to consider mathematically is that originally treated by Turing (1952), namely two coupled reactiondiffusion equations in which the interacting chemicals have different diffusion coefficients. For suitable reaction kinetics, as the ratio of diffusion coefficients increases from unity, for example, there is a critical value at which the uniform steady state becomes unstable to a particular spatial mode; this is a Turing bifurcation.

Early work on the Turing mechanism focussed on using linear analysis to predict the form of spatial patterns and their implications in a range of applications (Murray, 1981, 1982; Gierer and Meinhardt, 1972; Segel and Jackson, 1972). More recently, in parallel with continued work on applications (Meinhardt, 1993; Nagorcka, 1995a,b; Sherratt, 1995), there has been considerable interest in the mathematical nature of the Turing bifurcation. Following the majority of workers, we will consider this in the context of a square domain with zero flux (Neumann) boundary conditions. In this case, the uniform steady state may bifurcate to either a striped or a spotted type spatial pattern. In particular, when the primary bifurcation point is degenerate, there exist three distinct solution branches bifurcating from the uniform steady state, each of which represents two solutions that are identical except for having the opposite polarity. One represents striped solutions parallel to the $x$-axis, another striped solutions parallel to the $y$-axis and the final one represents spotted solutions with pattern in both the $x$ and the $y$ directions. When interpreted as patterns using a threshold level for pigmentation, for example, these solution branches correspond to stripes parallel to one pair of edges of the domain, stripes in the perpendicular direction, and a spotted pattern.

An important question concerning these multiple solution branches is their stability as reaction-diffusion solutions. Standard non-linear bifurcation analysis (Sattinger, 1972) shows that the selection of stripes versus spots is dependent on the non-linear terms in the reaction kinetics and therefore may not be predicted from the linearised model equations (Ermentrout 1991; Lyons and Harrison, 1991, 
1992). In particular, it can be shown that stable spotted and stable striped solutions cannot coexist (Ermentrout, 1991).

The degeneracy of the Turing bifurcation makes it difficult to study mathematically. One obvious approach to removing this degeneracy is to consider a rectangular rather than a square domain (see, for example, Maini et al., 1991, where this is done for a chemotactic pattern generator), which splits the bifurcation in a manner that we will discuss in detail later in the paper. However, our main focus is an alternative and rather less obvious method of splitting the bifurcation, namely to consider the case in which the diffusion coefficients are explicitly dependent on space, and vary slowly across the domain, parallel to one pair of edges. The explicit dependence of diffusion rates on space is important in some applications, including in particular the development of cartilage pattern in the embryonic chick limb (Maini et al., 1992; Benson et al., 1993), but in the present paper we will be concerned with the mathematical implications for the structure of the Turing bifurcation. We will show that the introduction of spatial variation in diffusivities provides a highly effective way of splitting the bifurcation, more effective than the use of a rectangular domain. We will use this technique to derive bifurcation diagrams in which the various solution branches are clearly separated, leading to a clear picture of the structure of the Turing bifurcation. In addition, we will show that a number of new phenomena are introduced by the spatial variation, including stable subcritical Turing patterns.

\section{Mathematical framework}

We investigate the effects of slow spatial variations in the diffusion coefficients of two interacting morphogens, with concentrations $u(x, y, t)$ and $v(x, y, t)$. We consider, in particular, spatially heterogeneous steady state solutions of the non-dimensionalised reaction diffusion system

$$
\begin{gathered}
\nabla \cdot(\mathscr{D}(x, \eta) \nabla u)+F(u, v)=\partial u / \partial t \\
\mu \nabla \cdot(\mathscr{D}(x, \eta) \nabla v)+G(u, v)=\partial v / \partial t
\end{gathered}
$$

defined on the unit square $0<x, y<1$ with zero flux boundary conditions. We consider the case in which $\mathscr{D}(x, \eta)$ is a function of the perturbation parameter $\eta$ and the spatial variable $x$, and to be specific we consider the case

$$
\mathscr{D}(x, \eta)=D+\eta x^{2}
$$


where $D$ is a positive constant. Crucially, $\partial \mathscr{D} / \partial x \rightarrow 0$ as $\eta \rightarrow 0$; otherwise there is no special significance in the form (2.1c), and we focus on a particular spatial dependence to enable explicit calculation of the various terms in the expansions near bifurcation points. In (2.1), $\nabla=\left(\begin{array}{l}\partial / \partial x \\ \partial / \partial y\end{array}\right)$. We take $\mu$, the ratio of diffusion coefficients, to be the bifurcation parameter. Standard linear analysis shows that, for particular kinetics $F$ and $G$ and with $\eta=0$, there is a critical value $\mu_{c}$ of $\mu$ at which the uniform steady state loses linear stability. When the uniform steady state is linearly unstable to a single mode, with unique wavenumber, standard bifurcation theory (Sattinger, 1972) shows that close to the bifurcation point there exists a family of analytic solutions of the form

$$
\begin{gathered}
\left(\begin{array}{l}
u(\underline{x}) \\
v(\underline{x})
\end{array}\right)=\left(\begin{array}{l}
u_{0} \\
v_{0}
\end{array}\right)+\varepsilon\left(\begin{array}{l}
u_{1}(\underline{x}) \\
v_{1}(\underline{x})
\end{array}\right)+\varepsilon^{2}\left(\begin{array}{l}
u_{2}(\underline{x}) \\
v_{2}(\underline{x})
\end{array}\right)+O\left(\varepsilon^{3}\right) \\
\mu=\mu_{c}+\varepsilon \tau_{1}+\varepsilon^{2} \tau_{2}+O\left(\varepsilon^{3}\right),
\end{gathered}
$$

where $\underline{x}=(x, y),\left(u_{0}, v_{0}\right)$ is the uniform steady state, and $|\varepsilon| \ll 1$. Substituting (2.2) into the full nonlinear system (2.1) and equating powers of $\varepsilon$ determines the functions $u_{i}, v_{i}$ and the values of $\tau_{i}$. This leads to an approximate solution of the weakly nonlinear problem (Iooss and Joseph, 1980).

Our work in this paper deals entirely with the particular spatial dependence (2.1c) for the diffusion coefficients. An important related problem is to consider the effects of small, irregular variations in diffusivity or domain geometry, such as would occur in any real biological system to which one might apply the Turing theory. Although the results we will describe have no formal implications for this case, they do suggest the intuitive possibility that such variations might have a significant effect on the bifurcation structure.

For small non-zero $\eta$, we will show that the bifurcation structure of the non-linear system may be found using a two parameter perturbation technique (Bauer et al., 1975), wherein primary (and certain secondary) solution branches are expressed as asymptotic expansions in the two small parameters, $\eta$ and $\varepsilon$.

In this case,

$$
\begin{gathered}
\left(\begin{array}{l}
u(\underline{\underline{x}}) \\
v(\underline{\underline{x}})
\end{array}\right)=\left(\begin{array}{l}
u_{0} \\
v_{0}
\end{array}\right)+\varepsilon\left(\begin{array}{l}
u_{1}(\underline{x}, \eta) \\
v_{1}(\underline{x}, \eta)
\end{array}\right)+\varepsilon^{2}\left(\begin{array}{l}
u_{2}(\underline{x}, \eta) \\
v_{2}(\underline{x}, \eta)
\end{array}\right)+O\left(\varepsilon^{3}\right) \\
\mu=\mu_{B}(\eta)+\varepsilon \tau_{1}(\eta)+\varepsilon^{2} \tau_{2}(\eta)+O\left(\varepsilon^{3}\right)
\end{gathered}
$$


where for each $i=1,2, \ldots$

$$
\begin{gathered}
\left(\begin{array}{l}
u_{i}(\underline{x}, \eta) \\
v_{i}(\underline{x}, \eta)
\end{array}\right)=\left(\begin{array}{l}
u_{i}^{0}(\underline{x}) \\
v_{i}^{0}(\underline{x})
\end{array}\right)+\eta\left(\begin{array}{l}
u_{i}^{1}(\underline{x}) \\
v_{i}^{1}(\underline{x})
\end{array}\right)+\eta^{2}\left(\begin{array}{l}
u_{i}^{2}(\underline{x}) \\
v_{i}^{2}(\underline{x})
\end{array}\right)+O\left(\eta^{3}\right) \\
\tau_{i}(\eta)=\tau_{i}^{0}+\eta \tau_{i}^{1}+\eta^{2} \tau_{i}^{2}+O\left(\eta^{3}\right)
\end{gathered}
$$

and $\mu_{B}(\eta)$ is the primary bifurcation point. For small non-zero $\eta$, we assume that $\mu_{B}(\eta)$ lies close to $\mu_{c}$ and may be expressed as a power series in $\eta$,

$$
\mu_{B}(\eta)=\mu_{c}+\eta w_{1}+\eta^{2} w_{2}+O\left(\eta^{3}\right) .
$$

Following standard techniques (Iooss and Joseph, 1980) we scale $\varepsilon$ such that

$$
\begin{array}{ll}
\left\|\left(u_{1}, v_{1}\right)\right\|=1, & \left\langle\left(u_{1}, v_{1}\right),\left(u_{2}, v_{2}\right)\right\rangle=0, \\
\left\|\left(u_{1}^{1}, v_{1}^{1}\right)\right\|=1, & \left\langle\left(u_{1}^{0}, v_{1}^{0}\right),\left(u_{1}^{1}, v_{1}^{1}\right)\right\rangle=0,
\end{array}
$$

to obtain a unique expression for each solution branch; here the inner product is taken as the integral over the unit square domain of the scalar product of the two vectors.

We will show that substituting these expansions into (2.1) and solving the resulting equations to leading order indicates a splitting of the degenerate bifurcation point $\mu_{c}$ when $\eta \neq 0$, giving two simple primary bifurcation points and a secondary bifurcation point, which lies on one of the primary solution branches. When $\eta$ is small the secondary bifurcation point, $\mu_{\mathbf{S}}(\eta)$, lies close to the primary bifurcation point and steady state solutions of the model equations (2.1) may be written in terms of their displacement from the primary solution branch. In particular, following Mahar and Matkowsky (1977), we write the solutions on the secondary branch (denoted $\bar{u}, \bar{v})$ as

$$
\begin{aligned}
\left(\begin{array}{l}
\bar{u}(\underline{x}, \delta, \eta) \\
\bar{v}(\underline{x}, \delta, \eta)
\end{array}\right) & =\left(\begin{array}{l}
u_{P}(\underline{x}, \eta) \\
v_{P}(\underline{x}, \eta)
\end{array}\right)+\delta\left(\begin{array}{l}
\bar{u}_{1}(\underline{x}, \eta) \\
\bar{v}_{1}(\underline{x}, \eta)
\end{array}\right)+\delta^{2}\left(\begin{array}{l}
\bar{u}_{2}(\underline{x}, \eta) \\
\bar{v}_{2}(\underline{x}, \eta)
\end{array}\right)+O\left(\delta^{3}\right) \\
\mu & =\mu_{S}(\eta)+\delta v_{1}(\eta)+\delta^{2} v_{2}(\eta)+O\left(\delta^{3}\right)
\end{aligned}
$$

where for each $i=1,2, \ldots$

$$
\begin{aligned}
\left(\begin{array}{l}
\bar{u}_{i}(\underline{x}, \eta) \\
\bar{v}_{i}(\underline{x}, \eta)
\end{array}\right) & =\eta^{1 / 2}\left(\begin{array}{l}
\bar{u}_{i}^{1}(\underline{x}) \\
\bar{v}_{i}^{1}(\underline{x})
\end{array}\right)+\eta\left(\begin{array}{l}
\bar{u}_{i}^{2}(\underline{x}) \\
\bar{v}_{i}^{2}(\underline{x})
\end{array}\right)+O\left(\eta^{3 / 2}\right), \\
v_{i}(\eta) & =v_{i}^{0}+\eta^{1 / 2} v_{i}^{1}+\eta v_{i}^{2}+O\left(\eta^{3 / 2}\right) .
\end{aligned}
$$

Here $\underline{u}_{P}(\underline{x}, \eta)$ denotes the solution vector on the primary bifurcation branch $\bar{h}$ at the point $\mu=\mu_{S}(\eta)$ at which the secondary bifurcation 
occurs. We assume that this secondary bifurcation point corresponds to a value $\varepsilon_{B}(\eta)$ of the parameter $\varepsilon$, which depends on $\eta$ according to the relation

$$
\varepsilon_{B}=\eta^{1 / 2} \varepsilon_{1}+\eta^{1} \varepsilon_{2}+O\left(\eta^{3 / 2}\right)
$$

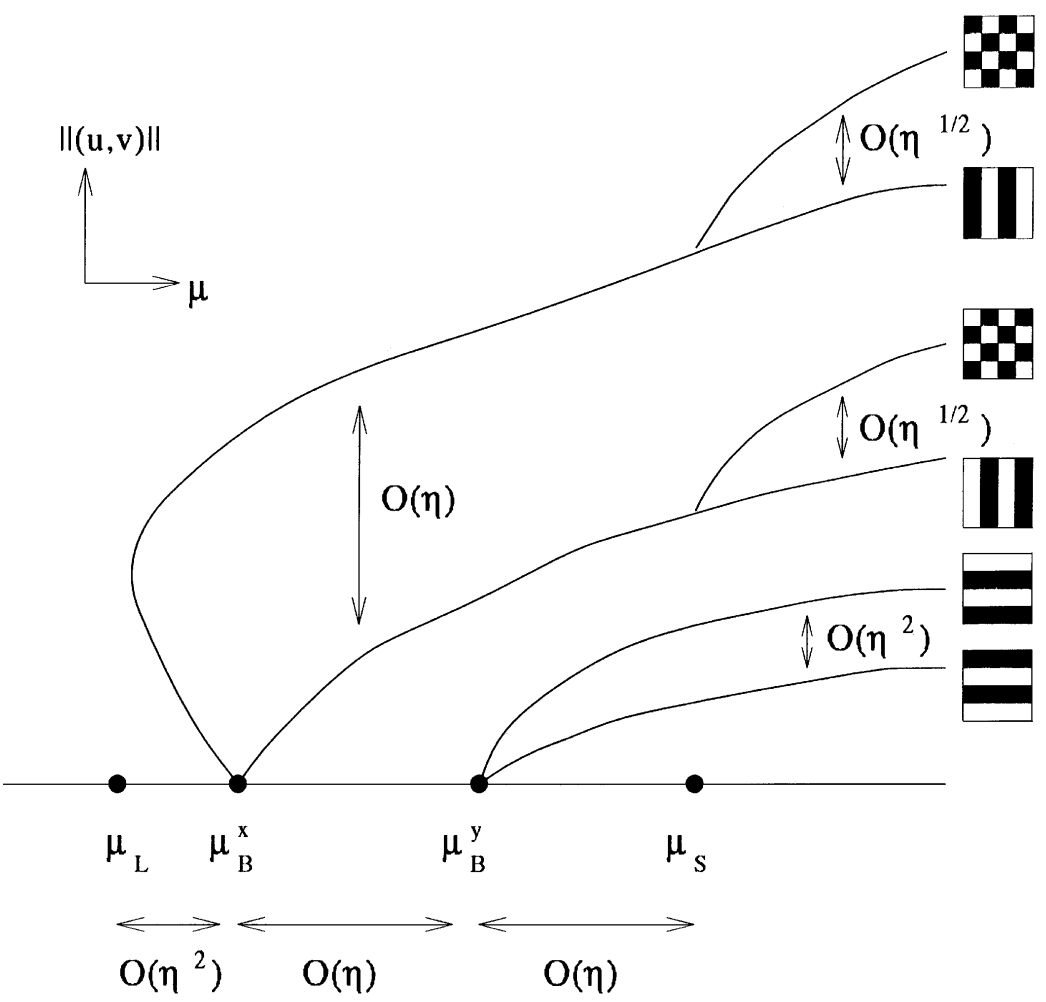

Fig. 1. A schematic representation of the bifurcation diagram for pattern formation in the system (2.1) when there is a small spatial variation in the diffusion coefficient, parameterised by $\eta \ll 1$. The form of this bifurcation diagram is derived in Sects. 3 and 4; stability, which is not shown in this figure, is discussed in Sect. 5. In the spatially homogeneous case $(\eta=0)$ the Turing bifurcation is highly degenerate; this figure illustrates how the various solution branches separate when spatial inhomogeneity is introduced. The bifurcation point splits into two separate primary bifurcation points, $\mu=\mu_{B}^{x}$ and $\mu=\mu_{B}^{y}$, and a secondary bifurcation point $\mu_{S}$. Moreover, the pairs of solution branches, which are coincident when $\eta=0$, separate for non-zero $\eta$. As illustrated schematically, the primary solution branches correspond to striped patterns, and the secondary solution branches correspond to spotted patterns. In these schematic illustrations of pattern type, the $x$-axis of the square domain runs across the page, and the $y$-axis runs up the page. On the figure, the dependence of the various branch separations on $\eta$ are indicated. These are all calculated in the text, although it should be noted that the separation of the two primary solution branches emerging from $\mu_{B}^{y}$ has not be determined beyond showing that there is no term that is $\gg \eta^{2}$. All the other separations have been determined so that the indicated separations are strict order of magnitude dependencies on $\eta$ 
where $\varepsilon_{1}, \varepsilon_{2}, \ldots$ are real and $\varepsilon_{1} \neq 0$. Here, without loss of generality, $\eta$ is assumed positive. In this case, the solutions (2.6) become power series expansions in the perturbation parameter $\eta$ and the small parameter $\delta$, which is a measure of the distance from the secondary bifurcation point. The unknowns, $\bar{u}_{i}^{j}, v_{i}^{j}$ and $\varepsilon_{i}$ may therefore be determined by substituting the power series expansions into the model equations and collecting terms of equal power in $\eta$ and $\delta$. The method of analysis is completely analogous to the determination of the primary solution branches.

Figure 1 shows a schematic representation of the bifurcation structure when $\eta \neq 0$. The stability of the various solutions in this structure can be determined in a standard way, and we will discuss the results of this study in Sect. 5.

To be specific, in the numerical simulations illustrated in this paper we will use the standard non-dimensionalised Schnakenberg kinetics (Schnakenberg, 1979)

$$
F(u, v)=\Gamma\left(A-u+v u^{2}\right) \text { and } G(u, v)=\Gamma\left(B-v u^{2}\right),
$$

where $\Gamma, A$ and $B$ are positive parameters. However, the analytical results are quite general.

\section{Primary solution branches}

Substituting the power series expansions (2.3) into the model equations (2.1) we first collect terms of $O(\varepsilon)$ and equate them to zero. For $\eta=0$, this leads to the linearised system for spatially homogeneous diffusion coefficients. We assume that this system admits a single unstable wavenumber, denoted by $k_{c}$. The general solution for $\left(u_{1}^{1}, v_{1}^{1}\right)$ (recalling the zero flux boundary conditions) may therefore be written in the form $\sum_{i^{2}+j^{2}=k_{\mathrm{c}}^{2}}\left(C_{i, j}^{u}, C_{i, j}^{v}\right) \cos (i \pi x) \cos (j \pi y)$ for some suitably defined constants $C_{i, j}^{\mathfrak{u}}$ and $C_{i, j}^{v}$. However, since the domain may be rescaled such that $k_{c}^{2}=1$ (Ermentrout, 1991) we assume that the first unstable wavenumbers are $(i=1, j=0)$ and $(i=0, j=1)$. Together with the normalisation condition, $\left|\left(u_{1}^{0}, v_{1}^{0}\right)\right|^{2}=1$, this implies that

where

$$
\left(\begin{array}{l}
u_{1}^{0} \\
v_{1}^{0}
\end{array}\right)=\left(\begin{array}{l}
1 \\
m
\end{array}\right) C_{x} \cos (\pi x)+C_{y} \cos (\pi y),
$$

$$
m=\frac{D \pi^{2}-F_{u}}{F_{v}}
$$

and

$$
C_{x}^{2}+C_{y}^{2}=C^{2} \equiv \frac{2}{1+m^{2}}
$$


Here $F_{u}$ denotes $\partial F /\left.\partial u\right|_{\left(u_{0}, v_{0}\right)}$ and we extend this notation in the obvious way, so that for example $G_{u v}$ denotes $\partial^{2} G /\left.\partial u \partial v\right|_{\left(u_{0}, v_{0}\right)}$.

Equating terms to $O(\eta)$ and using the Fredholm Alternative yields the solvability conditions

$$
\frac{w_{1} D m p \pi^{2}}{2} C_{x}+\left(1+\mu_{c} m p\right)\left(\frac{\pi^{2}}{6}-\frac{1}{4}\right) C_{x}=0,
$$

and

$$
\frac{w_{1} D m p \pi^{2}}{2} C_{y}+\left(1+\mu_{c} m p\right) \frac{\pi^{2}}{6} C_{y}=0,
$$

where $p=\left(D \pi^{2}-F_{u}\right) / G_{u}$. Recall that the constant $w_{1}$ appears in the expansion of $\mu_{B}(\eta)$, defined in equation (2.4). Thus, for a solution to exist, the unknowns $C_{x}, C_{y}$ and $w_{1}$ must satisfy either

$$
C_{x}^{2}=C^{2}, \quad C_{y}=0, \quad w_{1}=w_{1}^{x} \equiv-\frac{2\left(1+\mu_{c} m p\right)}{D m p \pi^{2}}\left(\frac{\pi^{2}}{6}-\frac{1}{4}\right)
$$

or

$$
C_{x}=0, \quad C_{y}^{2}=C^{2}, \quad w_{1}=w_{1}^{y} \equiv-\frac{2\left(1+\mu_{c} m p\right)}{D m p \pi^{2}}\left(\frac{\pi^{2}}{6}\right) .
$$

This implies that the degenerate bifurcation point for $\eta=0$, at $\mu=\mu_{c}$, has split into two simple primary bifurcation points given by

$$
\begin{aligned}
& \mu_{B}^{x}=\mu_{c}+\eta w_{1}^{x}+O\left(\eta^{2}\right) \\
& \mu_{B}^{y}=\mu_{c}+\eta w_{1}^{y}+O\left(\eta^{2}\right) .
\end{aligned}
$$

Note that the splitting of multiple primary bifurcation points in the presence of small non-zero parameters has been observed for a number of other systems (see, for example, Bauer et al. (1975) and Reiss (1983)). Hunding and Brøns (1990) also reported this phenomenon in their study of the effects of spatial variation in a three-dimensional reaction diffusion model of cell division during early embryonic development.

\subsection{The branch bifurcating at $\mu_{B}^{x}$}

We now proceed to determine leading order power series approximations to the primary solution branches bifurcating from each of $\mu_{B}^{x}$ and $\mu_{B}^{y}$. First we consider the family of solutions $\{(u(x, \varepsilon, \eta), v(x, \varepsilon, \eta))$, $\mu(\varepsilon, \eta)\}$ bifurcating from the primary bifurcation point $\mu_{B}^{x}$, defined in (3.5a). From equations (3.1) and (3.4a) it follows that

$$
\left(\begin{array}{l}
u_{1}^{0}(\underline{x}) \\
v_{1}^{0}(\underline{x})
\end{array}\right)=\left(\begin{array}{l}
1 \\
m
\end{array}\right) C \cos (\pi x)
$$


where $m$ and $C$ are defined by equations (3.2) and (3.3) respectively. We can then determine $\left(u_{1}^{1}, v_{1}^{1}\right)$ (see Appendix 1) and hence approximate the primary solution branch by

$$
\begin{aligned}
\left(\begin{array}{l}
u \\
v
\end{array}\right) & =\left(\begin{array}{l}
u_{0} \\
v_{0}
\end{array}\right)+\varepsilon\left[A\left(\begin{array}{l}
1 \\
m
\end{array}\right) \cos (\pi x)+\eta \sum_{i}\left(\begin{array}{l}
a_{i}^{u} \\
a_{i}^{v}
\end{array}\right) \cos (i \pi x)\right] \begin{array}{c}
\text { higher } \\
+ \text { order } \\
\text { terms }
\end{array} \\
\mu & =\mu_{B}^{x}(\eta)+\varepsilon \eta \tau_{1}^{1}+\varepsilon^{2} \tau_{2}^{0}+\text { higher order terms },
\end{aligned}
$$

where $a_{i}^{u}$ and $a_{i}^{v}(i=1,2, \ldots)$ are constants. Here our notation is as described in Sect. 2, with $\tau_{i}^{j}$ denoting the coefficient of $\varepsilon^{i} \eta^{j}$ in the expansion of $\mu$. In fact the parameter $\tau_{2}^{0}$ plays a key role in the bifurcation structure, and we will denote it henceforth as $\tau_{2}^{x}$, to distinguish it from the coefficient of $\varepsilon^{2}$ in another expansion, to be introduced later.

To leading order, the above approximation depends only on the spatial variable $x$. It may be shown by induction that the whole power series expansion is independent of the spatial variable $y$, and thus that the primary solution branch is quasi-one-dimensional. In particular, this implies that (3.6) are power series expansions for steady state solutions of the one-dimensional reaction diffusion system

$$
\begin{gathered}
\frac{\partial}{\partial x} \mathscr{D}(x, \eta) \frac{\partial u}{\partial x}+f(u, v)=\frac{\partial u}{\partial t} \\
\mu \frac{\partial}{\partial x} \mathscr{D}(x, \eta) \frac{\partial v}{\partial x}+g(u, v)=\frac{\partial v}{\partial t} .
\end{gathered}
$$

The solutions of (3.7) have spatially varying wavelengths of oscillation, and the asymmetry in wavelength increases with the spatial variation in $\mathscr{D}$ (Benson et al., 1993). Figure 2 compares the analytical approximations (3.6) for $u$ with numerical solutions of (3.7) for Schnakenberg kinetics (2.8).

There is good qualitative agreement between the solutions for all $|\eta|,|\varepsilon|<1$, but good quantitative agreement holds only when the system is close to the bifurcation point and $|\eta| \ll 1$. As expected, for values of $|\eta| \ll 1$, the variations in pattern wavelength and amplitude are small and are thus hard to detect in numerical simulation, but the spatial asymmetry becomes clearly visible as $\eta$ is increased.

Figure 2c compares the approximation for $u$ in two spatial dimensions. Since the primary solutions depend only on the spatial variable $x$, they predict spatial patterns which represent stripes perpendicular to 


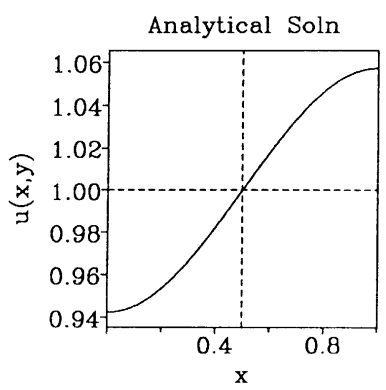

(a)
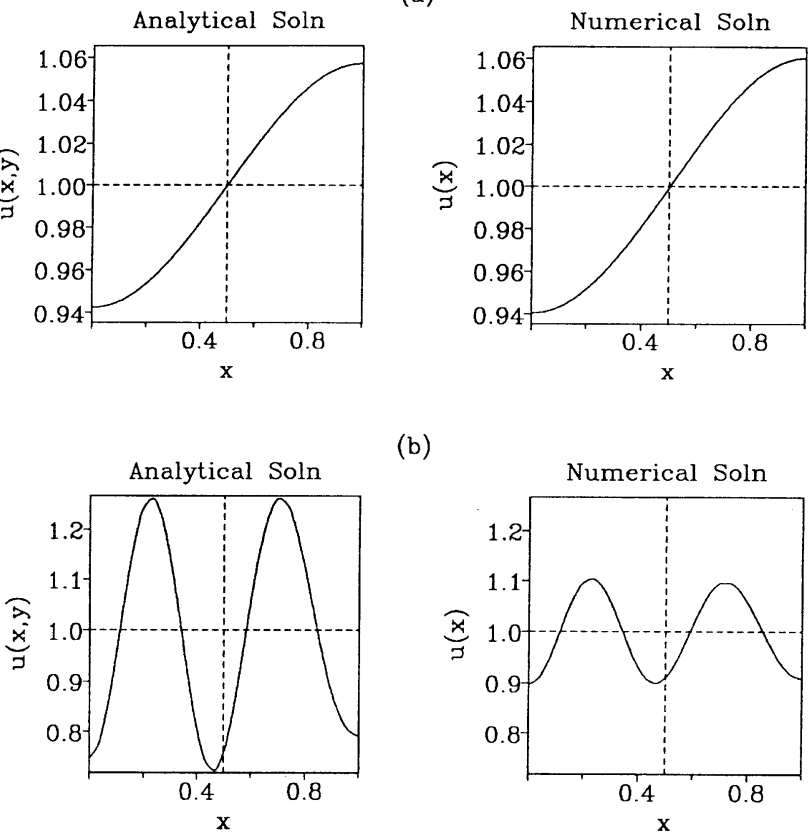

(b)

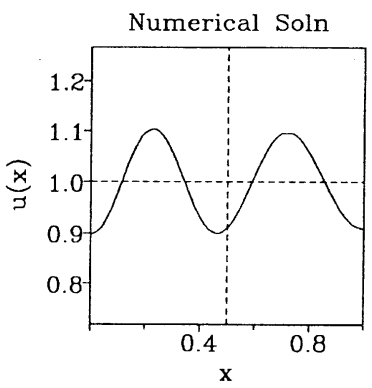

Fig. 2. A comparison of the truncated form of the power series approximations (3.6) with numerical solutions of (3.7) for Schnakenberg kinetics (2.8). The parameter values used were (a) $\eta=0.01, A=0.2, \quad B=0.8, \quad \Gamma=30, \mu=15, \quad D=1$ and (b) $\eta=$ $0.75 A=0.1, B=0.9, \Gamma=464, \mu=464, D=1$. For these values (a) $\mu_{B}^{x}=14.9252$, $k_{c}=1$ (as in the text) and (b) $\mu_{B}^{x}=8.5556, k_{c}=4$. The analytical solutions are plotted as functions of $x$ at an arbitrary value of $y$. The numerical solutions were found by solving (3.7) with the NAG routine D03PGF, which converts the equations to a system of coupled odes using the method of lines, and then solves these using Gear's method

the $x$ axis. Moreover, since pattern wavelength is asymmetric in the $x$ direction, the width of the stripes may vary within the domain; of course, this only applies when $k_{c}>1$, so that there is more than one stripe. The spatial variation in the width of the stripes is barely visible for $|\eta| \ll 1$ but becomes significant as $|\eta|$ increases.

Using the truncated power series solutions, it is possible to construct a bifurcation diagram of the primary solution branch bifurcating from $\mu_{B}^{x}$ for a given set of parameter values. The theoretical diagram is constructed by calculating the possible values $\varepsilon$ from the equation (3.6b) and substituting these values into the expression (3.6a). At each value of $\mu$, the real parameter $\varepsilon$ may assume one of the two values $\varepsilon^{+}$and $\varepsilon^{-}$, given by

$$
\varepsilon^{ \pm}=\frac{-\eta \tau_{1}^{1} \pm\left\{\left(\eta \tau_{1}^{1}\right)^{2}+4 \tau_{2}^{x}\left(\mu-\mu_{B}^{x}\right)\right\}^{1 / 2}}{2 \tau_{2}^{x}}
$$



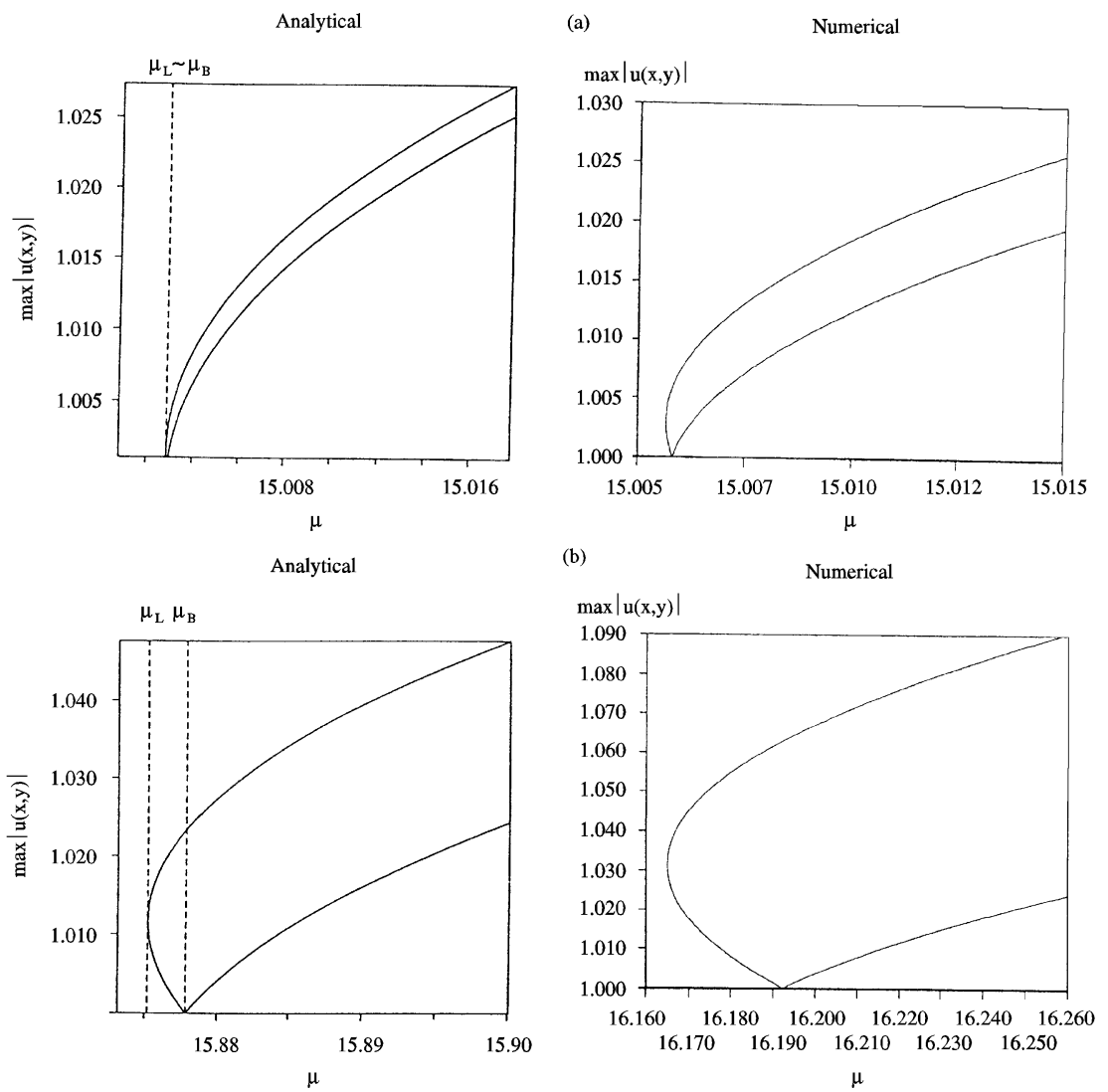

Fig. 3. A comparison of bifurcation diagrams constructed from the truncated power series approximations (left) from (3.6) with those produced by AUTO (right). The parameter values used are as in Fig. 2(a) with the exception that in (a) $\eta=0.05$ and (b) $\eta=0.5$. In this case, our analysis predicts that (a) $\mu_{B} \approx \mu_{L}$ with value 15.0028 , and in (b) $\mu_{B}=15.878, \mu_{L}=15.875$. In turn AUTO predicts that in (a) $\mu_{B} \approx \mu_{L}$ with value 15.006 and in (b) $\mu_{B}=16.194, \mu_{L}=16.1649$

Thus the power series expansion (3.6) represents two solutions $u\left(x, \varepsilon^{+}, \eta\right)$ and $u\left(x, \varepsilon^{-}, \eta\right)$ of different magnitude, and on the bifurcation diagram (Fig. 3), where we plot $\mu$ against the maximum value of $\|u(x, \varepsilon, \eta)\|$, there will appear two distinct arms of the primary solution branch. This separation of the branches when $\eta \neq 0$ represents an additional splitting of the Turing bifurcation in comparison to the case $\eta=0$, when the two branches are coincident, representing identical patterns but with opposite polarity. In practice, the difference between $\left\|\underline{u}\left(x, \varepsilon^{-}, \eta\right)\right\|$ and $\left\|u\left(x, \varepsilon^{+}, \eta\right)\right\|$ is barely visible at very small values of $\eta$, but as the magnitude of $\eta$ increases, the two arms of the primary solution branch may be distinguished in numerical simulations. 
When $\tau_{2}^{x}>0$, one arm is supercritical but the other has a small subcritical region. On this second arm, the point $\mu=\mu_{L}, \varepsilon=\varepsilon_{L}$, where $\mu_{L}=\mu_{B}-\left(\eta \tau_{1}^{1}\right)^{2} /\left(4 \tau_{2}^{x}\right)$ and $\varepsilon_{L}=-\eta \tau_{1}^{1} /\left(2 \tau_{2}^{x}\right)$, represents a simple limit point at which the branch changes direction. This implies that for each $\mu \in\left[\mu_{L}, \mu_{B}^{x}\right]$ there exist two subcritical solutions lying on the same arm of the primary solution branch, whilst for each $\mu>\mu_{B}^{x}$ there exist two supercritical solutions lying on different arms. A similar result holds when $\tau_{2}^{x}<0$. Thus, for $\eta \neq 0$, the uniform steady state undergoes a transcritical bifurcation at the point $\mu_{B}^{x}$. This contrasts to the case $\eta=0$, where the uniform steady state undergoes a pitchfork bifurcation, and primary solution branches represent stripes perpendicular to the $x$ axis which are supercritical when $\tau_{2}^{x}>0$ and subcritical when $\tau_{2}^{x}<0$ (see later).

Since the primary solution branch bifurcating from $\mu_{B}^{x}$ is quasi-onedimensional we may compare our theoretical bifurcation diagram with one produced by the numerical package AUTO (Doedel, 1986), which locates the bifurcation and limit points of a partial differential equation system defined in one spatial dimension and constructs the associated solution branches by a pseudo arc length continuation method. A comparison of the bifurcation diagrams is illustrated in Fig. 3, where the bifurcation parameter $\mu$ is plotted against the maximum amplitude of $\|\underline{u}(x, y)\|$. Again, there is good qualitative agreement for all $0<|\eta|<1$ and $0 \leqq|\varepsilon|<1$, but only good quantitative agreement when the system is close to the bifurcation point (i.e. $\varepsilon \ll 1$ and $|\eta| \ll 1$ ). In particular, these numerical results confirm that in contrast to the case $\eta=0$, there are stable subcritical striped solutions (Fig. 4). This is discussed in more detail in Sect. 5, when we consider the stability of the solution branches.

\subsection{The branch bifurcating at $\mu_{B}^{y}$}

Similar analysis can to used to calculate the power series expansions for the primary solution branch bifurcating from $\mu_{B}^{y}$ (see Appendix 1 for details) and we find that

$$
\begin{aligned}
\left(\begin{array}{l}
u \\
v
\end{array}\right)= & \left(\begin{array}{l}
u_{0} \\
v_{0}
\end{array}\right)+\varepsilon\left[A\left(\begin{array}{l}
1 \\
m
\end{array}\right) \cos (\pi y)\right. \\
& \left.+\eta \sum_{i}\left(\begin{array}{c}
b_{i}^{u} \\
b_{i}^{v}
\end{array}\right) \cos (i \pi x) \cos (\pi y)\right] \begin{array}{c}
\text { higher } \\
\text { order } \\
\text { terms }
\end{array} \\
\mu= & \mu_{B}^{y}(\eta)+\varepsilon^{2} \tau_{2}^{x}+\text { higher order terms },
\end{aligned}
$$





Fig. 4. An example of a subcritical stable steady solution of (2.1) represented in one and two spatial dimensions. The solution on the right was obtained by numerical solution of (3.7), on the interval [0,1], with the NAG routine D03PGF. The solution on the left was found by solving the full non-linear reaction diffusion system (2.1) on the unit square using a finite difference scheme to obtain a system of algebraic equations which were solved by an alternating direction implicit (ADI) method. The analysis presented in Appendix 2 predicts that this pattern is stable. In this example, Schnakenberg kinetics were used with parameter values as in Fig. 1(a) except that $\eta=1.2$ and $\mu=19.06$. AUTO predicts $\mu_{B}=19.2443$ and $\mu_{L}=18.9987$. Since the uniform steady state is also stable in this parameter range, initial conditions close to the theoretically predicted pattern were used to generate the solution

where $b_{i}^{u}, b_{i}^{v}(i=1,2, \ldots)$ are constants. Again we replace the coefficient $\tau_{2}^{0}$ of $\varepsilon^{2}$ by $\tau_{2}^{x}$, since detailed calculation (see Appendix 1) shows that the coefficient of $\varepsilon^{2}$ here is the same as that in the expansion (3.6b) at $\mu_{B}^{x}$, namely $\tau_{2}^{x}$, whose value is given in (A.5) in Appendix 1. For $\eta \neq 0$ it is seen that the power series expansion is dependent on both spatial variables and, in general, has no planes of symmetry. However, the $x$-dependence lies in a $O(\varepsilon \eta)$ term, that is of a lower order of magnitude than the $x$-independent $O(\varepsilon)$ term as $\eta \rightarrow 0$. Therefore, the truncated form of the power series approximation predicts stripes perpendicular to the $y$ axis whose spatial variation is $O(\eta)$ as $\eta \rightarrow 0$. Typical examples are illustrated in Fig. 5.

As before, there is good qualitative agreement between the solutions for all $0<|\eta|<1$ and $0 \leqq|\varepsilon|<1$, but the quantitative agreement is best close to the bifurcation point $(\varepsilon \ll 1)$, when $|\eta| \ll 1$. In this case, the asymmetries introduced into the wavelength and amplitude are small and barely visible in simulations. As the magnitude of $\eta$ increases, however, significant spatial asymmetry may be observed in the spatial patterns. Again, the pattern variation is captured by the truncated solutions in this case, since the terms $\varepsilon \eta \underline{u}_{1}^{1}$ and $\varepsilon \underline{u}_{1}^{0}$ are comparable.

Using (3.9), the bifurcation diagram for the primary solution branch bifurcating from $\mu_{B}^{v}$ may also be constructed. For each value of 
(a)



(b)

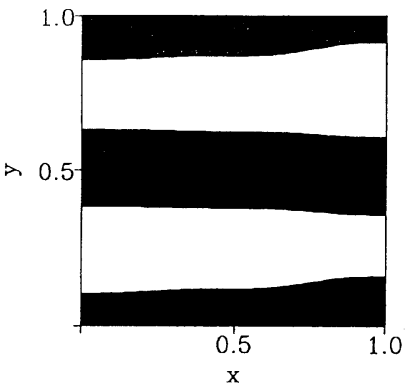

Fig. 5. Typical spatial patterns predicted by the truncated power series approximations when the threshold concentration is chosen to be $u(x, y)=u_{0}$. The parameter values used were (a) $A=0.2, B=0.8, \quad \Gamma=30, \quad \mu=15, \quad D=1, \quad \eta=0.05$ and (b) $A=0.1, \quad B=0.9, \quad \Gamma=464, \quad \mu=9, \quad D=1, \quad \eta=0.5$. For these values (a) $\mu_{B}^{y}=14.9286, k_{c}=1$ and (b) $\mu_{B}^{y}=8.5558, k_{c}=4$ (see text for details)

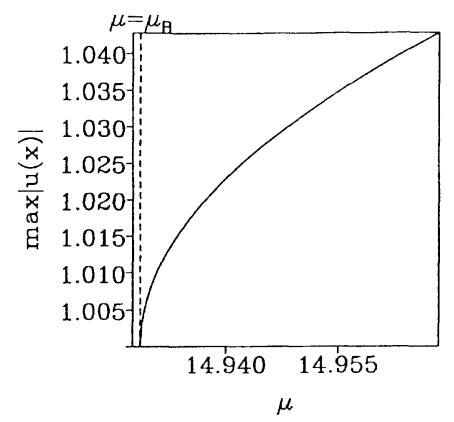

Fig. 6. Bifurcation diagram constructed from the truncated power series approximations (3.9). The parameter values used are as in Fig. 5(a). Our analysis predicts that $\mu_{B}^{v}=14.9286$. The infinite series in $i$ was calculated by summing the first 20 terms, on the basis that the next 20 terms make a negligible additional contribution

$\mu, \varepsilon$ takes one of the two values $\varepsilon^{ \pm}= \pm \sqrt{\left(\mu-\mu_{B}^{y}\right) / \tau_{2}^{x}}$, and thus the power series approximations represent two distinct solutions, $\underline{u}\left(x, \varepsilon^{+}, \eta\right)$ and $u\left(x, \varepsilon^{-}, \eta\right)$. However, these two solutions differ only by $\overline{\mathrm{a}}$ phase difference to first order, and thus on the bifurcation diagram (Figure 6) where we plot $\mu$ against the maximum of $\|\underline{u}(x, \varepsilon, \eta)\|$, the two branches are represented by a single curve. Note the contrast between this present case, where the two primary branches differ only at $O\left(\varepsilon^{2}\right)$, and the bifurcation at $\mu_{B}^{x}$, where the two bifurcating branches differ at $O(\varepsilon)$. For $\tau_{2}^{x}>0$ the solution branches are supercritical and for $\tau_{2}^{x}<0$ they are subcritical (cf. the primary solution branch bifurcating from $\mu_{B}^{x}$ ). Figure 6 illustrates our theoretical bifurcation diagram for a given set of parameters. 


\subsection{Primary bifurcations when $\eta=0$}

To compare the primary bifurcation structure when $\eta \neq 0$ with that when $\eta=0$, that is, the case of spatially homogeneous coefficients, we have used the above technique to show that when $\eta=0$ there are three distinct primary solution branches bifurcating from $\mu_{c}$ of the form:

$$
\begin{aligned}
\left(\begin{array}{l}
u \\
v
\end{array}\right)= & \left(\begin{array}{l}
u_{0} \\
v_{0}
\end{array}\right)+\varepsilon\left(\begin{array}{l}
1 \\
m
\end{array}\right) C \cos (\pi x) \\
& +\varepsilon^{2}\left\{\left(\begin{array}{l}
\alpha_{u} \\
\alpha_{v}
\end{array}\right) \frac{\kappa C^{2} \cos (2 \pi x)}{2}+\left(\begin{array}{l}
\beta_{u} \\
\beta_{v}
\end{array}\right) \frac{\kappa C^{2}}{2}\right\}+O\left(\varepsilon^{3}\right) \\
\mu & =\mu_{c}+\varepsilon^{2} \tau_{2}^{x}+O\left(\varepsilon^{3}\right),
\end{aligned}
$$

which represents stripes perpendicular to the $x$ axis,

$$
\begin{aligned}
\left(\begin{array}{l}
u \\
v
\end{array}\right)= & \left(\begin{array}{l}
u_{0} \\
v_{0}
\end{array}\right)+\varepsilon\left(\begin{array}{l}
1 \\
m
\end{array}\right) C \cos (\pi y) \\
& +\varepsilon^{2}\left\{\left(\begin{array}{l}
\alpha_{u} \\
\alpha_{v}
\end{array}\right) \frac{\kappa C^{2} \cos (2 \pi y)}{2}+\left(\begin{array}{c}
\beta_{u} \\
\beta_{v}
\end{array}\right) \frac{\kappa C^{2}}{2}\right\}+O\left(\varepsilon^{3}\right) \\
\mu= & \mu_{c}+\varepsilon^{2} \tau_{2}^{x}+O\left(\varepsilon^{3}\right),
\end{aligned}
$$

which represents stripes perpendicular to the $y$ axis, and

$$
\begin{aligned}
\left(\begin{array}{l}
u \\
v
\end{array}\right)= & \left.\left(\begin{array}{l}
u_{0} \\
v_{0}
\end{array}\right)+\varepsilon\left(\begin{array}{l}
1 \\
m
\end{array}\right) C_{x} \cos (\pi x)+C_{y} \cos (\pi y)\right) \\
& +\varepsilon^{2}\left\{\left(\begin{array}{l}
\alpha_{u} \\
\alpha_{v}
\end{array}\right) \frac{\kappa\left(C_{x}^{2} \cos (2 \pi x)+C_{y}^{2} \cos (2 \pi y)\right)}{2}\right. \\
& \left.+\left(\begin{array}{c}
\beta_{u} \\
\beta_{v}
\end{array}\right) \frac{\kappa\left(C_{x}^{2}+C_{y}^{2}\right)}{2}+\left(\begin{array}{l}
\gamma_{u} \\
\gamma_{v}
\end{array}\right) C_{x} C_{y} \cos (\pi x) \cos (\pi y)\right\}+O\left(\varepsilon^{3}\right) \\
\mu= & \mu_{c}+\varepsilon^{2} \tau_{2}^{x y}+O\left(\varepsilon^{3}\right)
\end{aligned}
$$

$\left(C_{y}^{2}+C_{x}^{2}=C^{2}\right)$ which represents spotted solutions. Here $\kappa=$ $\frac{1}{2} F_{u u}+F_{u v}$, and the constants $\alpha_{u}, \alpha_{v}, \beta_{u}, \beta_{v}, \gamma_{u}$ and $\gamma_{v}$ are defined in Appendix 1. Recall that, without loss of generality, we assume that $k_{c}=1$. Strictly speaking the last power series expansion represents two 
distinct solution branches, one for which $C_{x}=C_{y}$ and the other for which $C_{x}=-C_{y}$. The parameter $\tau_{2}^{x y}$, which is in general non-zero, is defined by the equation

$$
\begin{aligned}
& \frac{D m p \pi^{2} \tau_{2}^{x y}}{2}+(-1+p) \kappa \frac{C^{2}}{2}\left(F_{u u}+m F_{u v}\right)\left(\frac{\alpha_{u}}{8}+\frac{\beta_{u}}{2}+\frac{\gamma_{u}}{2}\right) \\
& +(-1+p) \kappa \frac{C^{2}}{2} F_{u v}\left(\frac{\alpha_{v}}{8}+\frac{\beta_{v}}{2}+\frac{\gamma_{v}}{2}\right)+(-1+p) F_{u u v} \frac{9 C^{2}}{16}=0
\end{aligned}
$$

The power series expansions above are in agreement with the well known fact that, in the case of constant diffusion coefficients and zero flux boundary conditions, the uniform steady state undergoes a pitchfork bifurcation. In particular, striped solution branches are supercritical when $\tau_{2}^{x}>0$ and subcritical when $\tau_{2}^{x}<0$, whilst the spotted solution branches are supercritical when $\tau_{2}^{x y}>0$ and subcritical when $\tau_{2}^{x y}<0$. Thus, in contrast to the case $\eta \neq 0$, there are no transcritical bifurcations.

Comparing equations (3.6) and (3.10a), it is clear that the solution branch bifurcating from $\mu_{B}^{x}$ when $\eta \neq 0$ represents a perturbation from the striped solution branch perpendicular to the $x$ axis when $\eta=0$. Similarly, the solution branch (3.9) bifurcating from $\mu_{B}^{y}$ when $\eta \neq 0$ represents a perturbation from the striped solution branch (3.10b) perpendicular to the $y$ axis when $\eta=0$. Moreover, our analysis shows in detail how the different bifurcation points and branches collapse as $\eta \rightarrow 0$ to give the degenerate structure described above (see Fig. 1). However, when $\eta \neq 0$, there is no primary solution branch analogous to the spotted solution branch (3.10c) when $\eta=0$. In the following section we show that this is due to a further splitting of the primary bifurcation point so that spots arise as secondary bifurcations from primary striped solution branches.

\section{Secondary bifurcations}

In this section we determine secondary bifurcations of the model system (2.1) which occur close to the primary bifurcation points $\mu_{B}^{x}$ and $\mu_{B}^{y}$ using the method outlined in Sect. 2. We recall from (2.6) our notation that $\underline{u}_{P}(x, \eta)$ represents the solution on the primary solution branch at the point at which a secondary bifurcation occurs, namely $\mu=\mu_{S}(\eta)$ or equivalently $\varepsilon=\varepsilon_{B}(\eta)$. When the secondary bifurcation point lies on the primary solution branch bifurcating from $\mu_{B}^{x}$, the 
solution is

$$
\begin{aligned}
\left(\begin{array}{l}
u_{P}^{x}(\eta) \\
v_{P}^{x}(\eta)
\end{array}\right)= & \left(\begin{array}{l}
u_{0} \\
v_{0}
\end{array}\right)+\eta^{1 / 2}\left(\begin{array}{c}
1 \\
m
\end{array}\right) C \varepsilon_{1} \cos (\pi x) \\
& +\eta\left(\begin{array}{l}
\alpha_{u} \\
\alpha_{v}
\end{array}\right) \frac{\kappa C^{2} \varepsilon_{1}^{2} \cos (2 \pi x)}{2}+\eta\left(\begin{array}{l}
\gamma_{u} \\
\gamma_{v}
\end{array}\right) \frac{\kappa C^{2} \varepsilon_{1}^{2}}{2} \\
& +\eta\left(\begin{array}{c}
1 \\
m
\end{array}\right) C \varepsilon_{2} \cos (\pi x)+O\left(\eta^{3 / 2}\right)
\end{aligned}
$$

with

$$
\mu_{S}^{x}(\eta)=\mu_{c}+\eta\left(w_{1}^{x}+\tau_{2}^{x} \varepsilon_{1}^{2}\right)+O\left(\eta^{3 / 2}\right),
$$

and when it lies on the solution branch bifurcating from $\mu_{B}^{y}$, it is

$$
\begin{aligned}
\left(\begin{array}{c}
u_{P}^{y}(\eta) \\
v_{P}^{y}(\eta)
\end{array}\right)= & \left(\begin{array}{l}
u_{0} \\
v_{0}
\end{array}\right)+\eta^{1 / 2}\left(\begin{array}{c}
1 \\
m
\end{array}\right) C \varepsilon_{1} \cos (\pi y)+\eta\left(\begin{array}{c}
\alpha_{u} \\
\alpha_{v}
\end{array}\right) \frac{\kappa C^{2} \varepsilon_{1}^{2} \cos (2 \pi y)}{2} \\
& +\eta\left(\begin{array}{l}
\gamma_{u} \\
\gamma_{v}
\end{array}\right) \frac{\kappa C^{2} \varepsilon_{1}^{2}}{2}+\eta\left(\begin{array}{c}
1 \\
m
\end{array}\right) C \varepsilon_{2} \cos (\pi y)+O\left(\eta^{3 / 2}\right)
\end{aligned}
$$

with

$$
\mu_{S}(\eta)=\mu_{c}+\eta\left(w_{1}^{y}+\tau_{2}^{x} \varepsilon_{1}^{2}\right)+O\left(\eta^{3 / 2}\right) .
$$

Here the constants $\varepsilon_{1}$ and $\varepsilon_{2}$ are coefficients of powers of $\eta^{1 / 2}$ in the expansion (2.7) of $\varepsilon_{B}(\eta)$; these constants remain to be determined. The various other constants were defined during the determination of the primary solution branch (Sect. 3 and Appendix 1).

In the power series expansion (2.6) of the secondary solution branch, the variable $\delta$ is analogous to the parameter $\varepsilon$ along the primary solution branches. In particular, $\delta$ provides a measure of the distance from $\mu_{S}$ and is scaled such that

$$
\left\|\left(\bar{u}_{1}^{1}, \bar{v}_{1}^{1}\right)\right\|=1, \quad\left\langle\left(\bar{u}_{1}^{1}, \bar{v}_{1}^{1}\right),\left(\bar{u}_{2}^{1}, \bar{v}_{1}^{1}\right)\right\rangle=0 .
$$

With this choice of scaling, however, $\delta$ is singular at $\eta=0$. Thus, in contrast to the power series expansions for the primary solution branches, analytical approximations of the form (2.6) only exist for non-zero $\eta$. This reflects the fact that for $\eta=0$, secondary bifurcations do not generally occur close to the primary bifurcation point, so that secondary solution branches may not be written in terms of their displacement from a small amplitude primary solution, as in the power series expansions (2.6). There may be other choices for the parameterisation of the secondary solution branches which are non-singular as $\eta \rightarrow 0$, but for $\eta \neq 0$, the $\delta$ and $\eta$ expansion (2.6) is the most natural parameterisation of the secondary solution branch, and is thus the one we use. 
First we consider the secondary bifurcation points which lie along one of the primary solution branches bifurcating from $\mu_{B}^{x}$. At $O\left(\delta \eta^{1 / 2}\right)$, we have

$$
\left(\begin{array}{c}
\bar{u}_{1}^{1} \\
\bar{v}_{1}^{1}
\end{array}\right)=\left(\begin{array}{l}
1 \\
m
\end{array}\right)\left(C_{x} \cos (\pi x)+C_{y} \cos (\pi y)\right),
$$

where $\left(C_{x}^{2}+C_{y}^{2}\right)=C^{2}=2 /\left(1+m^{2}\right)$.

To $O(\eta)$, the Fredholm Alternative forces $v_{1}^{1}=0$. In this case

$$
\begin{aligned}
\left(\begin{array}{c}
\bar{u}_{1}^{2} \\
\bar{v}_{1}^{2}
\end{array}\right)= & \left(\begin{array}{c}
\alpha_{u} \\
\alpha_{v}
\end{array}\right) \frac{2 \kappa C \varepsilon_{1} C_{x} \cos (2 \pi x)}{2}+\left(\begin{array}{c}
\beta_{u} \\
\beta_{v}
\end{array}\right) \frac{2 \kappa C \varepsilon_{1} C_{x}}{2} \\
& +\left(\begin{array}{l}
\gamma_{u} \\
\gamma_{v}
\end{array}\right) 2 \kappa C \varepsilon_{1} C_{y} \cos (\pi x) \cos (\pi y) .
\end{aligned}
$$

The value of $\varepsilon_{1}$ may now be determined by collecting the terms at $O\left(\eta^{3 / 2}\right)$. The solvability conditions become (after much tedious algebra)

$$
\begin{aligned}
C_{x} & \frac{D m p \pi^{2}\left(w_{1}^{x}+\tau_{2}^{x} \varepsilon_{1}^{2}\right)}{2}+C_{x}\left(1+\mu_{c} m p\right)\left(\frac{\pi^{2}}{6}-\frac{1}{4}\right) \\
& +C_{x}(-1+p) \kappa C^{2} \varepsilon_{1}^{2} F_{u v}\left(\frac{3 \alpha_{v}}{8}+\frac{3 \beta_{v}}{4}\right) \\
& +C_{x}(-1+p) \kappa\left(C^{2} \varepsilon_{1}^{2}\left(F_{u u}+F_{u v} m\right)\left(\frac{3 \alpha_{u}}{8}+\frac{3 \beta_{u}}{4}\right)\right) \\
& +C_{x}(-1+p) \frac{9 F_{u u v} m C^{2} \varepsilon_{1}^{2}}{16}+C_{x} D m p \pi^{2} v_{1}^{2} C \varepsilon_{1}=0,
\end{aligned}
$$

and

$$
\begin{aligned}
C_{y} & \frac{D m p \pi^{2}\left(w_{1}^{x}+\tau_{2}^{x} \varepsilon_{1}^{2}\right)}{2}+C_{y} \frac{\left(1+\mu_{c} m p\right) \pi^{2}}{6} \\
& +C_{y}(-1+p) \kappa C^{2} \varepsilon_{1}^{2} F_{u v}\left(\frac{\beta_{v}}{4}+\frac{\gamma_{v}}{2}\right) \\
& +C_{y}(-1+p) \kappa C^{2} \varepsilon_{1}^{2}\left(F_{u u}+F_{u v} m\right)\left(\frac{\beta_{u}}{4}+\frac{\gamma_{u}}{2}\right) \\
& +C_{y}(-1+p) \frac{3 F_{u u v} m C^{2} \varepsilon_{1}^{2}}{8}=0 .
\end{aligned}
$$

From these equations we deduce that either

$$
C_{x}^{2}=C^{2}, \quad C_{y}=0, \quad v_{1}^{2}=2 \varepsilon_{1} \tau_{2}^{x},
$$


or

$$
C_{x}=0, \quad C_{y}^{2}=C^{2}, \quad v_{1}^{2}=0
$$

and

$$
\begin{gathered}
\varepsilon_{1}^{2}\left\{(-1+p)\left[C^{2}\left(F_{u u}+F_{u v} m\right)\left(\frac{\beta_{u}}{4}+\frac{\gamma_{u}}{2}\right)+C^{2} F_{u v}\left(\frac{\beta_{v}}{4}+\frac{\gamma_{v}}{2}\right)\right]\right. \\
\left.+\frac{3 C^{2} F_{u u v}}{8}+\frac{D m p \pi^{2} \tau_{2}^{x}}{2}\right\}+\frac{D m p \pi^{2} w_{1}^{x}}{2}+\frac{\left(1+\mu_{c} m p\right) \pi^{2}}{6}=0 .
\end{gathered}
$$

In the former case (4.5), the value of $\varepsilon_{1}$ is undetermined. This is a trivial case, in which the corresponding power series expansion (2.6) represents the continuation of the primary solution branch beyond the secondary bifurcation point, and may be obtained by replacing $\varepsilon$ by $\varepsilon_{1}+\delta \eta^{1 / 2}$ in our original analytical approximation of the primary solution branch.

To determine the secondary bifurcations, we must therefore consider (4.6). In this case there exists a secondary bifurcation point of the form (2.7) whenever equation (4.6) admits a real, non-zero solution for $\varepsilon_{1}$. This requires $\frac{\left(w_{1}^{x}-w_{1}^{y}\right)}{2\left(\tau_{2}^{x y}-\tau_{2}^{x}\right)}>0$, where $\tau_{2}^{x y}$ is defined by the equation (3.11). When this condition is satisfied

$$
\varepsilon_{1}=\varepsilon_{x}= \pm \sqrt{\frac{\left(w_{1}^{x}-w_{1}^{y}\right)}{2\left(\tau_{2}^{x y}-\tau_{2}^{x}\right)}}
$$

and equation (2.7) defines two distinct secondary bifurcation points. Moreover, since the corresponding values of $\varepsilon$ have opposite signs, these points lie on separate arms of the primary solution branch, but at the same value of $\mu$ (at least to this order), namely

$$
\mu=\mu_{S}^{x}(\eta) \equiv \mu_{c}+\eta\left(w_{1}^{x}+\frac{\tau_{2}^{x}\left(w_{1}^{x}-w_{1}^{y}\right)}{2\left(\tau_{2}^{x y}-\tau_{2}^{x}\right)}\right)
$$

By a similar analysis, we find that two secondary bifurcation points of the form (2.7) can occur on the primary solution branch bifurcating from $\mu_{B}^{y}$. In this case, however, we require $\frac{\left(w_{1}^{x}-w_{1}^{y}\right)}{2\left(\tau_{2}^{x y}-\tau_{2}^{x}\right)}<0$. Again both secondary bifurcations occur on separate primary solution branches at the same value of $\mu$ to leading order, which is

$$
\mu_{S}^{y}(\eta)=\mu_{c}+\eta\left(w_{1}^{y}+\frac{\tau_{2}^{x}\left(w_{1}^{y}-w_{1}^{x}\right)}{2\left(\tau_{2}^{x y}-\tau_{2}^{x}\right)}\right) .
$$

Thus a secondary bifurcation of the form (2.7) occurs on exactly one of the primary solution branches. Specifically, the secondary bifurcation points lie on the primary solution branch bifurcating from $\mu_{B}^{x}$ when 
$\frac{\left(w_{1}^{x}-w_{1}^{y}\right)}{2\left(\tau_{2}^{y y}-\tau_{2}^{x}\right)}>0$ and on the primary solution branch bifurcating from $\mu_{B}^{y}$ when $\frac{\left(w_{1}^{x}-w_{1}^{y}\right)}{2\left(\tau_{2}^{y}-\tau_{2}^{\chi}\right)}<0$. Each of these cases are therefore considered separately.

\subsection{Solutions on the secondary branches}

We can determine the leading order approximations to the secondary solution branches bifurcating from $\mu_{S}^{x}$ and $\mu_{S}^{v}$. We find, after a great deal of tedious algebra, that the power series expansion for the secondary solution branch bifurcating from $\mu_{S}^{x}$ is given by

$$
\begin{aligned}
\left(\begin{array}{l}
u \\
v
\end{array}\right)= & \left(\begin{array}{l}
u_{0} \\
v_{0}
\end{array}\right)+\eta^{1 / 2}\left(\begin{array}{l}
1 \\
m
\end{array}\right) C \varepsilon_{x} \cos (\pi x) \\
& +\eta\left(\begin{array}{l}
\alpha_{u} \\
\alpha_{v}
\end{array}\right) \frac{\kappa C^{2} \varepsilon_{x}^{2} \cos (2 \pi x)}{2}+\eta\left(\begin{array}{l}
\beta_{u} \\
\beta_{v}
\end{array}\right) \frac{\kappa C^{2} \varepsilon_{x}^{2}}{2}+O\left(\eta^{3 / 2}\right) \\
& +\delta\left\{\eta^{1 / 2}\left(\begin{array}{l}
1 \\
m
\end{array}\right) C \cos (\pi y)+\eta\left(\begin{array}{l}
\gamma_{u} \\
\gamma_{v}
\end{array}\right) \kappa 2 C^{2} \varepsilon_{x} \cos (\pi x) \cos (\pi y)+O\left(\eta^{3 / 2}\right)\right\} \\
& +\delta^{2}\left\{\eta^{1 / 2}\left(\begin{array}{l}
1 \\
m
\end{array}\right) \frac{C \cos (\pi x)}{2 \varepsilon_{x}}+\eta\left(\begin{array}{l}
\alpha_{u} \\
\alpha_{v}
\end{array}\right) \kappa C^{2} \frac{\cos (2 \pi x)+\cos (2 \pi y)}{2}\right. \\
& \left.+\eta\left(\begin{array}{l}
\beta_{u} \\
\beta_{v}
\end{array}\right) \kappa C^{2} \varepsilon_{x}^{2}+O\left(\eta^{3 / 2}\right)\right\}+O\left(\delta^{3}\right) \\
\mu= & \mu_{c}+\left(w_{1}^{x}+\tau_{2}^{x} \varepsilon_{x}^{2}\right) \eta+\delta^{2} \eta 2 \tau_{2}^{x y}+O\left(\delta^{3}\right) .
\end{aligned}
$$

By a similar analysis we may determine the secondary solution branches bifurcating from $\mu_{S}^{y}$. In this case the power series expansions have the form

$$
\begin{aligned}
\left(\begin{array}{l}
u \\
v
\end{array}\right)= & \left(\begin{array}{l}
u_{0} \\
v_{0}
\end{array}\right)+\eta^{1 / 2}\left(\begin{array}{l}
1 \\
m
\end{array}\right) C \varepsilon_{y} \cos (\pi y) \\
& +\eta\left\{\left(\begin{array}{l}
\alpha_{u} \\
\alpha_{v}
\end{array}\right) \kappa \frac{C^{2} \varepsilon_{y}^{2} \cos (2 \pi y)}{2}+\left(\begin{array}{l}
\beta_{u} \\
\beta_{v}
\end{array}\right) \kappa \frac{C^{2} \varepsilon_{y}^{2}}{2}\right\}+O\left(\eta^{3 / 2}\right) \\
& +\delta\left\{\eta^{1 / 2}\left(\begin{array}{l}
1 \\
m
\end{array}\right) C \cos (\pi x)+\eta\left(\begin{array}{l}
\gamma_{u} \\
\gamma_{v}
\end{array}\right) 2 \kappa C^{2} \varepsilon_{y} \cos (\pi x) \cos (\pi y)+O\left(\eta^{3 / 2}\right)\right\} \\
& +\delta^{2}\left\{\eta^{1 / 2}\left(\begin{array}{l}
1 \\
m
\end{array}\right) \frac{C \cos (\pi y)}{2 \varepsilon_{y}}+\eta\left(\begin{array}{l}
\alpha_{u} \\
\alpha_{v}
\end{array}\right) \kappa C^{2} \frac{\cos (2 \pi x)+\cos (2 \pi y)}{2}\right. \\
& \left.+\eta\left(\begin{array}{l}
\beta_{u} \\
\beta_{v}
\end{array}\right) \kappa C^{2}+O\left(\eta^{3 / 2}\right)\right\}+O\left(\delta^{3}\right)
\end{aligned}
$$


(a)

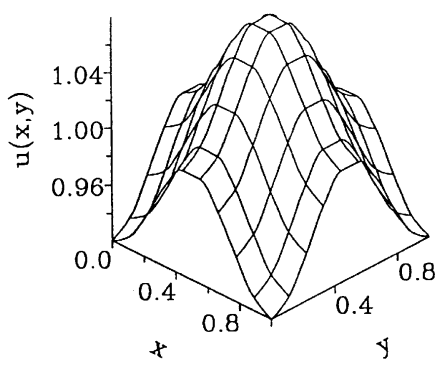

(b)



Fig. 7. A comparison of (a) the truncated form of the power series approximations (4.11) with (b) numerical solutions of the Schnackenberg model with $\eta=0.35$. The parameter values used were $A=0.2, B=0.8, \Gamma=120, \mu=15.74$ and $D=1$. In this case, $\mu_{B}^{s}=14.9323$ and $k_{c}=2$

$$
\mu=\mu_{c}+\left(w_{1}^{y}+\tau_{2}^{x} \varepsilon_{y}^{2}\right) \eta+\delta^{2} \eta 2 \tau_{2}^{x y}+O\left(\delta^{3} \eta\right)
$$

where

$$
\varepsilon_{y}= \pm \sqrt{\frac{\left(w_{1}^{y}-w_{1}^{x}\right)}{2\left(\tau_{2}^{x y}-\tau_{2}^{x}\right)}}
$$

In both cases the power series expansions show clearly the expected two-dimensional nature of the steady state solutions which lie on the secondary solution branches. To compare these analytical expansions with numerically computed solutions of the full non-linear system, we use the power series truncated at $O\left(\eta^{1 / 2} \delta\right)$.

Figure 7 compares these truncated expansions for the concentration profile of $u$ with numerical solutions of the full non-linear system (2.1). As expected, we find that, for the parameter values considered, there is reasonable qualitative agreement between the solutions for all $0<|\eta|<1$ and $0 \leqq|\delta|<1$, but good quantitative agreement only when the system is close to the secondary bifurcation point and $\eta \ll 1$. In this case, the spatial patterns are dependent almost exclusively on a single spatial variable. As the magnitudes of $|\delta|, \eta \rightarrow 1$, however, pattern becomes discernible in both directions. Figure 8 illustrates typical two-dimensional spatial patterns predicted by our analytical approximations. These patterns are the analogue of the spotted solutions generated by reaction diffusion systems with spatially homogeneous diffusion coefficients. In this case, however, the spotted solutions are asymmetric; the asymmetry being more clearly visible as $\eta$ increases. Using the truncated power series solutions, it is possible to construct a bifurcation diagram of the secondary solution branches 
(a)



(b)

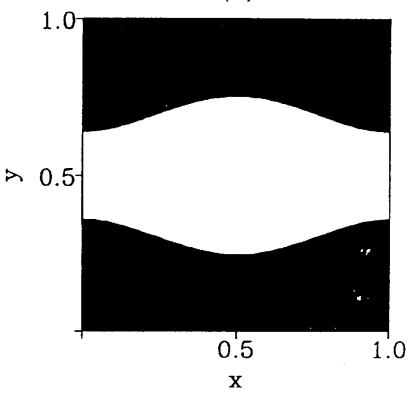

Fig. 8. Spatial patterns predicted by the truncated power series approximations (4.11) when the threshold concentration is chosen to be $u(x, y)=u_{0}$. The parameter values used were (a) $A=0.2, B=0.8, \Gamma=30, \mu=17.1, D=1, \eta=0.75$, and (b) $A=0.1$, $B=0.9, \Gamma=30, \mu=8.57, D=1, \eta=0.05$. In (a) $k_{c}=1, \mu_{S}=\mu_{S}^{x}=16.6461$ and in (b) $k_{c}=2, \mu_{S}=\mu_{S}^{x}=8.5677$

Table 1. A comparison of the truncated power series approximation for the secondary bifurcation point $\mu_{S}^{x}$ with the values of the primary bifurcation points predicted by our analysis. The truncated analytic approximations $\mu_{S}^{x}=\mu_{c}+\eta\left(w_{1}^{x}+\varepsilon_{x} \tau_{2}^{x}\right), \mu_{B}^{x}=\mu_{c}+\eta w_{1}^{x}$ and $\mu_{S}^{x}=\mu_{c}+\eta w_{1}^{y}$ are used, with parameter values as in Fig. 9a

\begin{tabular}{llll}
\hline$\eta$ & $\mu_{\mathrm{S}}^{x}$ & $\mu_{\mathrm{B}}^{x}$ & $\mu_{B}^{y}$ \\
\hline 0.0 & - & 14.9056 & 14.9056 \\
0.01 & 14.9323 & 14.9251 & 14.9286 \\
0.05 & 15.0391 & 15.0029 & 15.0203 \\
0.1 & 15.1725 & 15.10011 & 15.1349 \\
0.5 & 16.2402 & 15.8669 & 16.0521 \\
1.0 & 17.5746 & 16.8500 & 17.1985 \\
\hline
\end{tabular}

bifurcating from $\mu_{S}^{x}$ and $\mu_{S}^{y}$. Table 1 lists typical values of $\mu_{S}^{x}$, $\mu_{B}^{x}$ and $\mu_{B}^{y}$ predicted by our analysis.

On the bifurcation diagram (Fig. 9), where we plot $\mu$ against the maximum value of $\|u(\underline{x})\|$, the pairs of both primary and secondary solutions are represented by a single branch, because the value of $\eta$ is sufficiently small that the difference between solution arms are not visible. These diagrams should be compared with the schematic representation in Fig. 1. Note that the truncated power series approximations predict a pitchfork bifurcation from either $\mu_{S}^{x}$ or $\mu_{S}^{y}$. For $\tau_{2}^{x y}>0$ the secondary solution branches are supercritical and for $\tau_{2}^{x y}<0$ they are subcritical. 


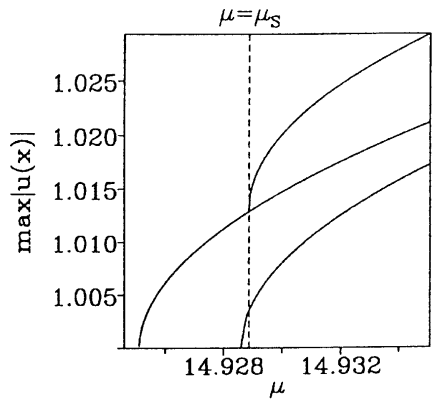

Fig. 9. Bifurcation diagram constructed from the truncated power series approximations showing primary and secondary solution branches. (For clarity only one primary solution branch is illustrated.) The parameter values used are as in Fig. 9(a) with the exception that $\eta=0.01$. In this case the secondary bifurcation points lie on the primary solution branches bifurcating from $\mu_{B}^{x}$, and has value $\mu_{S}^{x}=14.9323$. Our analysis predicts that $\mu_{B}^{x}=14.9252, \mu_{B}^{y}=14.9286$

\section{Stability}

It is straightforward, although algebraically complex, to determine the stability of the various solution branches. Details of the calculations are given in Appendix 2; here we summarise the results and discuss their implications. The key parameters controlling stability are $\tau_{2}^{x}$ and $\tau_{2}^{x y}$, and the various stability regions in the $\tau_{2}^{x}-\tau_{2}^{x y}$ plane are shown in Fig. 10. These results have some important implications for the possible range of observable (stable) patterns:

(i) The subcritical striped patterns, which are a novel feature introduced by spatially varying diffusion, can be stable. Specifically, this occurs for stripes perpendicular to the $x$-axis (on a primary branch bifurcating from $\mu_{x}^{B}$ ) when $\mu_{\mathrm{L}}<\mu_{B}^{x}$ and $\tau_{2}^{x}>0$.

(ii) Since $\tau_{2}^{x}>0$ is exactly the criterion for the primary branches bifurcating at $\mu_{B}^{y}$ to be supercritical, only supercritical striped patterns perpendicular to the $y$-axis are stable.

(iii) The primary solution branch on which the secondary bifurcation to spots occurs can change stability at this secondary bifurcation point; this occurs when $\tau_{2}^{x y}>\tau_{2}^{x}>0$, on whichever primary branch the secondary bifurcation to spots occurs.

(iv) The secondary solution branch (corresponding to spots) is never stable when it is subcritical, so that stable spots can only appear supercritically.

These observations together mean that stable stripes can appear either subcritically or supercritically, and then bifurcate supercritically to stable spots. Moreover, stable striped and spotted patterns never co-exist. This last observation has a direct analogy in the spatially homogeneous case $\eta=0$, in which either a striped or spotted solution (but never both) is selected at the (primary) Turing bifurcation point (Ermentrout, 1991; Lyons and Harrison, 1991).

For the small values of $\varepsilon, \eta$ and $\delta$ for which our weakly nonlinear analysis is valid, the range of subcritical values of the bifurcation 


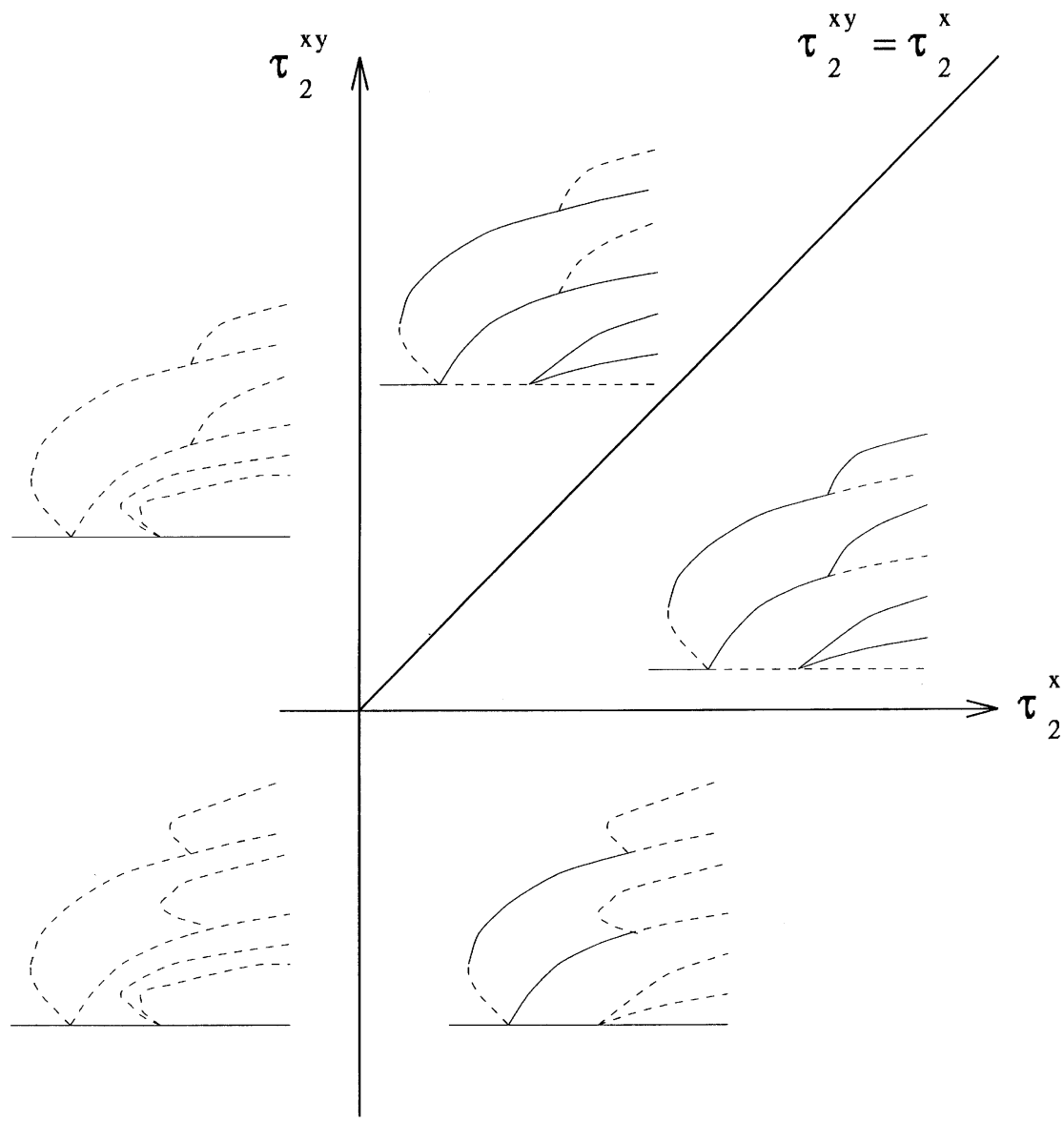

Fig. 10. A schematic representation of the stability of the various primary and secondary solution branches, as predicted by the analysis in Appendix 2. The four bifurcation diagrams all have the basic structure shown in Fig. 1; stable solutions are denoted by solid lines, and unstable solutions by dashed lines. This representation applies when the secondary bifurcation occurs on the primary solution branches emerging from $\mu=\mu_{B}^{x}$. In the alternative case in which the secondary bifurcation occurs on the primary branch emerging from $\mu=\mu_{B}^{v}$, the only difference is that the stability of the these two sets of primary branches is reversed. There are four stability regions in the $\tau_{2}^{x}-\tau_{2}^{x y}$ plane: (i) $\tau_{2}^{x}<0$ in which all patterns are unstable; (ii) $\tau_{2}^{x}>0, \tau_{2}^{x y}<0$ in which case the only stable patterns are on the primary branch between the primary and secondary bifurcations; (iii) $\tau_{2}^{x y}>\tau_{2}^{x}>0$ in which case the primary solution branches are stable and the secondary branches are unstable; (iv) $\tau_{2}^{x}>\tau_{2}^{x y}>0$ in which case the primary branches emerging from $\mu=\mu_{B}^{y}$ are stable, while those emerging from $\mu=\mu_{B}^{x}$ are initially unstable, and then lose stability at the secondary bifurcation, giving stable spotted solutions. Note that $\tau_{2}^{x}<0$ is exactly the condition for (both) solution branches emerging from $\mu=\mu_{B}^{y}$ to be subcritical, these solution branches change stability and criticality simultaneously, as illustrated 
(a)

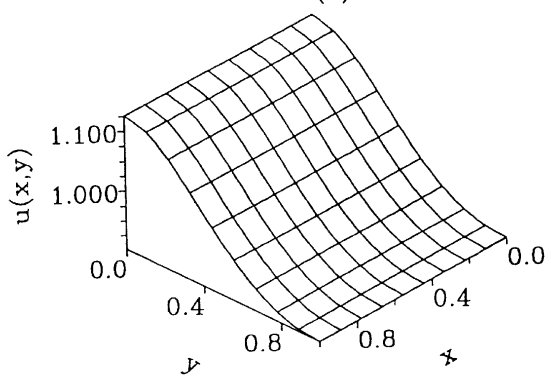

(b)

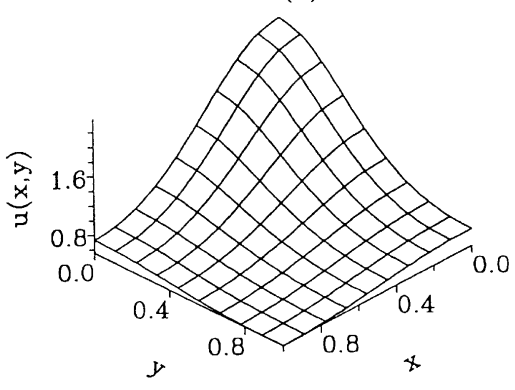

Fig. 11. Steady state solutions of the full non-linear system (2.1) for parameter values $\Gamma=30, \eta=1.2, A=0.2, B=0.8$, (a) $\mu=19.06$ and (b) $\mu=21.06$. In this case, our analysis predicts that the secondary bifurcation point, $\mu_{S}^{x}$, lies on the primary solution branch bifurcating from $\mu_{B}^{x}$. From AUTO, $\mu_{B}^{x}=19.244$ and $\mu_{L}=18.988$. The parameter values used satisfy the stability conditions $\tau_{2}^{x}>\tau_{2}^{x y}>0$. Note that the striped solution is subcritical, and for other initial conditions from those used (perturbations about the spatially heterogeneous steady state in each case) the system relaxed to the uniform steady state

parameter for which stable stripes exist is numerically very small. Similarly, the secondary bifurcation points lie in very close proximity to the primary bifurcation point. It is therefore difficult to investigate quantitatively our stability predictions by numerical simulation, because of the long relaxation time of solutions close to the bifurcation points. For larger values of $\eta$, the subcritical part of the primary solution branch is wider, and intuitively we would expect the position of the secondary bifurcation point to move further from the primary bifurcation point. In this case, therefore, it may be possible to observe a transition from stable striped to stable spotted solutions as the value of the bifurcation parameter is increased. Preliminary simulations suggest that this is true, but we have not done a detailed numerical study.

Figure 11 illustrates solutions of the full time dependent non-linear model equations solved numerically for $\eta \neq 0$ with parameter values satisfying $\tau_{2}^{x}>\tau_{2}^{x y}>0$. In this example we use a relatively large value of $\eta(>1)$, but nevertheless we find stable striped solutions close to the primary bifurcation point (predicted numerically from the corresponding one-dimensional system using AUTO) and stable spotted solutions for larger values of the bifurcation parameter. In this case, however, the truncated power series approximation cannot make an accurate prediction of the secondary bifurcation point. A higher order approximation may provide a better prediction for large values of $\eta$, although it must be remembered that the assumptions underlying the power series approximation are not valid. 
We complete this section by comparing the stability of striped and spotted solutions of the reaction diffusion system (2.1) for $\eta \neq 0$ with the stability of similar solutions in the spatially homogeneous case $\eta=0$. When the diffusion coefficients are constant, a similar analysis to that in Appendix 2 may be used to determine the stability of the primary solution branches bifurcating from $\mu_{c}$, via power series expansions (2.2). In this case we find that primary striped solutions are stable if and only if $\tau_{2}^{x y}>\tau_{2}^{x}>0$, and that primary spotted solutions are stable if and only if $\tau_{2}^{x}>\tau_{2}^{x y}>0$. These conditions are a statement of the well known fact that for constant diffusion the primary solution branches are stable only when the pitchfork bifurcation from the uniform steady is supercritical. Moreover, they agree with recent analyses which show that simultaneously stable striped and spotted solutions cannot exist (Ermentrout, 1991; Lyons and Harrison, 1991). It may also be shown that, when the model equations are written in terms of displacement from the uniform steady state, the absence of quadratic terms forces $\left(\tau_{2}^{x y}-\tau_{2}^{x}\right)<0$. Our stability conditions are therefore in agreement with the fact that certain kinetics exclude stable spotted solutions.

Moreover, the conditions for stable spotted solutions are the same for both $\eta=0$ and $\eta \neq 0$. The conditions for the stability of striped solutions when $\eta \neq 0$, however, are the same as for constant diffusion only beyond the secondary bifurcation point. In this case, the spatial pattern selected for either $\eta=0$ or $\eta \neq 0$ is determined by the competition between cubic and quadratic terms in the kinetics. For values of the bifurcation parameter less than the secondary bifurcation value, however, a striped solution is always selected. Thus, a reaction diffusion system, which for a given set of parameter values and constant diffusion coefficients admits only stable spotted solutions, can be forced to generate stable striped solutions by spatially heterogeneous diffusion coefficients. Numerical simulations demonstrate that this may also occur when reaction kinetics are spatially heterogeneous or when the shape of the domain is rectangular (Lyons and Harrison, 1992).

\section{Discussion}

In this paper we have considered only square shaped domains. For constant diffusion coefficients this ensures that no predescribed asymmetry is imposed on the reaction diffusion system which might influence pattern selection. Analytically we have shown, however, that a small spatial asymmetry in diffusion coefficients is sufficient to 
exclude the formation of spotted solutions close to a primary bifurcation point. This results from the splitting of the primary bifurcation point, which for constant diffusion is highly degenerate, into two simple primary bifurcation points and a secondary bifurcation point. In this respect the bifurcation structure of reaction diffusion systems defined on square domains with spatially heterogeneous diffusion coefficients is similar to that of systems defined on rectangular domains with constant diffusion coefficients. In the latter case, striped steady state solutions parallel to the $x$ and $y$ axes generally bifurcate from distinct primary bifurcation points. On an almost square domain we have found that the two primary bifurcation points lie close together and spotted solutions appear as secondary bifurcations from one of the primary solution branches. In contrast to the case of spatially heterogeneous diffusion coefficients, however, there are no transcritical bifurcations from the uniform steady state and hence no stable striped solutions for subcritical values of the bifurcation parameter. This implies that the two systems are not isomorphic. Moreover, the four primary bifurcation branches only separate into two identical pairs on an almost square domain, whereas with spatially varying diffusion coefficients, all four primary branches become distinct. Thus the introduction of a small spatial variation in the diffusion coefficients is a more effective way of splitting the degeneracies in the Turing bifurcation.

Reaction diffusion systems with constant diffusion coefficients defined on infinite domains do not in general have stable subcritical striped solutions. In this case, there are three basic patterns which tessellate the plane and appear as steady state solutions of reaction diffusion systems. These are stripes, rhombs (which correspond to our spotted solutions) and hexagons (which are not possible on a rectangular or square domain). According to stability analysis (see, for example, Malomed and Tribel'skii, 1987), hexagonal patterns should appear first via a subcritical bifurcation. These patterns are stable but become unstable at a supercritical value of the bifurcation parameter. From the same primary bifurcation point, stripes bifurcate supercritically. These are unstable close to the bifurcation point, but become stable at large values of the bifurcation parameter. There is a region of bistability where both stripes and hexagons are stable. As on the finite domain, stable spots can form in place of stable stripes.

These theoretical predictions have been confirmed numerically for the Schnakenberg and Brussellator models (Dufiet and Boissonade, 1992a,b; De Wit et al., 1992). Our analysis suggests, however, that spatially heterogeneous diffusion coefficients could enable striped solutions to appear subcritically on an infinite domain. Although both 
numerical (Borcksman et al., 1992) and theoretical analyses (Walgraef and Schiller, 1987) have been carried out on systems with spatially inhomogeneous model parameters defined on infinite domains, to our knowledge, subcritical striped solutions have not been reported. Recently, however, such solutions were observed (Jensen et al., 1993) in numerical solutions of a reaction diffusion system, defined on a spatially homogeneous domain, in which the instability interval of a time periodic, spatially oscillating solution overlapped with that of a Turing pattern. The same system also produced spatial patterns which could be localised within the domain. In both cases, the spatial patterns were constant in time for a wide range of parameter values. In general, however, when a Turing instability and a time oscillatory instability occur simultaneously, the solutions are complex, spatially inhomogeneous, non-stationary structures (Perraud et al., 1993), which may be observed experimentally. This has led to the suggestion that by continuously varying the value of a single parameter, one might move from stationary Turing patterns, through spatio-temporal structures, to travelling excitable waves (Boissonade, 1994). In particular, this highlights the fact that reaction diffusion mechanisms have a far greater capacity for pattern formation than is suggested by the linear analysis of standard Turing systems.

\section{Appendix 1}

In this Appendix, we summarise the analytical determination of the primary solution branches, supplying various details that were omitted in Sect. 3.

\section{A1.1 The branch bifurcating at $\mu_{B}^{x}$}

Substituting the expansion (2.3b) for $\mu$ into the governing reaction diffusion equations (2.1) and equating powers of $\varepsilon \eta$, we find that $\left(u_{1}^{1}, v_{1}^{1}\right)$ is a solution of the equation

$$
\begin{aligned}
L\left(\begin{array}{c}
u_{1}^{1}(\underline{x}) \\
v_{1}^{1}(\underline{x})
\end{array}\right)= & x^{2}\left(\begin{array}{c}
1 \\
\mu_{c} m
\end{array}\right) C \pi^{2} \cos (\pi x)+2 x\left(\begin{array}{c}
1 \\
\mu_{c} m
\end{array}\right) C \pi \sin (\pi x) \\
& +D w_{1}^{x}\left(\begin{array}{c}
0 \\
m
\end{array}\right) C \pi^{2} \cos (\pi x),
\end{aligned}
$$

where $L$ is the linearised operator for the nonlinear system (2.1). Recall that the parameters $m, C$ and $w_{1}^{x}$ are defined in (3.2), (3.3) and (3.4) 
respectively; $\mu_{c}$ is the value of $\mu$ at which a Turing bifurcation occurs when $\eta=0$.

To determine the solution we express $\left(u_{1}^{1}, v_{1}^{1}\right)$ as the Fourier cosine expansion

$$
\left(\begin{array}{l}
u_{1}^{1}(\underline{x}) \\
v_{1}^{1}(\underline{x})
\end{array}\right)=\sum_{k, j \in \mathbb{N}} \underline{a}_{k, j} \cos (k \pi x) \cos (j \pi y) .
$$

Substituting this expression into equation (A.1), multiplying both sides by $\cos (k \pi x) \cos (j \pi y)$ and integrating over $[0,1] \times[0,1]$ gives, for each $k, j \in \mathbb{N}$

$$
M_{k, j}\left(\begin{array}{c}
a_{k, j}^{1} \\
a_{k, j}^{2}
\end{array}\right)=2\left(\underline{J}_{1}+\underline{J}_{2}+\underline{J}_{3}\right)
$$

where $\underline{J}_{1}, \underline{J}_{2}, \underline{J}_{3}$ are given by

$$
\begin{aligned}
\underline{J}_{1} & =\int C \pi^{2}\left(\begin{array}{c}
1 \\
\mu_{c} m
\end{array}\right) x^{2} \cos (\pi x) \cos (k \pi x) \cos (j \pi y) \mathrm{d} x \mathrm{~d} y \\
& =C\left(\begin{array}{c}
1 \\
\mu_{c} m
\end{array}\right)\left\{\begin{array}{cc}
2\left(1+k^{2}\right) /\left(1-k^{2}\right)^{2} & k \neq 1, k \text { odd }, j=0 \\
-2\left(1+k^{2}\right) /\left(1-k^{2}\right)^{2} & k \neq 1, k \text { even, } j=0 \\
\pi^{2}\left[1 / 6+1 / 4 \pi^{2}\right] & k=1, j=0 \\
0 & k=0, j \neq 0
\end{array}\right. \\
\underline{J}_{2} & =\int C \pi\left(\begin{array}{c}
1 \\
\mu_{c} m
\end{array}\right) x \sin (\pi x) \cos (k \pi x) \cos (j \pi y) d x d y \\
& =C\left(\begin{array}{c}
1 \\
\mu_{c} m
\end{array}\right)\left\{\begin{array}{cc}
-2 /\left(1-k^{2}\right) & k \neq 1, k \text { odd }, j=0 \\
2 /\left(1-k^{2}\right) & k \neq 1, k \text { even, } j=0 \\
-1 / 2 & k=1, j=0 \\
0 & k=0, j \neq 0
\end{array}\right. \\
\underline{J}_{3} & =\int D w_{1} C \pi^{2}\left(\begin{array}{c}
0 \\
m
\end{array}\right) \cos (\pi x) \cos (k \pi x) \cos (j \pi y) d x d y \\
& =C\left(\begin{array}{c}
0 \\
m
\end{array}\right)\left\{\begin{array}{rr}
D w_{1} \pi^{2} / 2 & k=1, j=0 \\
0 & k \neq 1, j=0 \\
0 & k=0, j \neq 0
\end{array}\right.
\end{aligned}
$$

Here $M_{k, j}$ for $k, j=1,2, \ldots$ are the matrices associated with the action of $L$ on an arbitrary vector of the form $(u, v) \propto \cos (k \pi x)$ 
$\times \cos (j \pi y)$, and are defined by

$$
M_{k, j}=\left(\begin{array}{cc}
-D\left(k^{2}+j^{2}\right) \pi^{2}+F_{u} & F_{v} \\
G_{u} & -\mu_{c} D\left(k^{2}+j^{2}\right) \pi^{2}+G_{v}
\end{array}\right) .
$$

For $(k, j) \neq(1,0)$ or $(0,1), \underline{a}_{k, j}=2 M_{k, j}^{-1}\left(J_{1}+\underline{J}_{2}+\underline{J}_{3}\right)$, since the matrices $M_{k, j}$ are invertible. In this case, $\underline{a}_{0, j}=\underline{0}$ and in general, $\underline{a}_{k, 0} \neq \underline{0}$. For $(k, j)=(1,0)$ or $(0,1), M_{k, j}$ is singular. In this case $\underline{a}_{k, j}$ can be written in the form

$$
\underline{a}_{k, j}=\mathscr{A}_{k, j}\left(\begin{array}{c}
1 \\
m
\end{array}\right)+\mathscr{B}_{k, j}\left(\begin{array}{c}
1 \\
-1 / m
\end{array}\right) .
$$

For $(k, j)=(1,0), \mathscr{A}_{k, j}$ must have the value 0 to satisfy the orthogonality condition $(2.5 \mathrm{~b})$ and $\mathscr{B}_{k, j}$ may be calculated from $\mathscr{B}_{k, j} M_{k, j}(1,-1 / m)=2\left(\underline{J}_{1}+\underline{J}_{2}+\underline{J}_{3}\right)$. For $(k, j)=(0,1)$, it is easily seen that $\mathscr{B}_{k, j}=0$ and that $\overline{\mathscr{A}}_{k, j}$ is undetermined. Considering higher order expansions, however, it may be shown that $\mathscr{A}_{0,1}=0$. Thus we find

$$
\left(\begin{array}{l}
u_{1}^{1}(\underline{x}) \\
v_{1}^{1}(\underline{x})
\end{array}\right)=\sum_{k} \underline{a}_{k} \cos (k \pi x)
$$

where $a_{k}=a_{k, 0}$. Note that this leading order power series approximation is completely independent of the spatial variable $y$.

To determine the leading order approximation for $\mu$ we must consider higher order terms in the expansions. Equating coefficients of $O\left(\varepsilon^{2} \eta^{0}\right)$ and using the Fredholm Alternative, we find that $\tau_{1}^{0}=0$ and

$$
\begin{aligned}
\left(\begin{array}{c}
u_{2}^{0} \\
v_{2}^{0}
\end{array}\right)= & \left(\begin{array}{c}
\alpha_{u} \\
\alpha_{v}
\end{array}\right) \frac{1}{2} \kappa C^{2} \cos (2 \pi x)+\left(\begin{array}{c}
\beta_{u} \\
\beta_{v}
\end{array}\right) \frac{1}{2} \kappa C^{2}+\left(\begin{array}{c}
1 \\
m
\end{array}\right) P \cos (\pi x) \\
& +\left(\begin{array}{c}
1 \\
m
\end{array}\right) Q \cos (\pi y) .
\end{aligned}
$$

Here $P=0$, in order to satisfy the orthogonality condition $(2.5 b)$, and $Q$ is still to be determined; as stated in the main text, $\kappa=\frac{1}{2} F_{u u}+F_{u v}$. The constants $\alpha_{u}, \alpha_{v}, \beta_{u}$ and $\beta_{v}$ are defined by the equations

$$
M_{2,0}\left(\begin{array}{c}
\alpha_{u} \\
\alpha_{v}
\end{array}\right)=\left(\begin{array}{c}
-1 \\
1
\end{array}\right), \quad M_{0,0}\left(\begin{array}{c}
\beta_{u} \\
\beta_{v}
\end{array}\right)=\left(\begin{array}{c}
-1 \\
1
\end{array}\right) .
$$

In a similar way, the constants $\gamma_{u}$ and $\gamma_{v}$ that are used in the main text are defined by

$$
M_{1,1}\left(\begin{array}{l}
\gamma_{u} \\
\gamma_{v}
\end{array}\right)=\left(\begin{array}{c}
-1 \\
1
\end{array}\right)
$$


Substituting this expression into the $O\left(\varepsilon^{2} \eta\right)$ equations yields the solvability conditions $Q=0$ and $K_{1}+K_{2}+K_{3}+K_{4}=0$, where

$$
\begin{aligned}
K_{1}= & \int C\left(F_{u u} a_{2}^{1}+F_{u v}\left(a_{2}^{2}+m a_{2}^{1}\right)\right)\left(\begin{array}{c}
-1 \\
1
\end{array}\right) \cdot\left(\begin{array}{l}
1 \\
p
\end{array}\right) \cos (2 \pi x) \cos ^{2}(\pi x) d x \\
& +\int C\left(F_{u u} a_{2}^{1}+F_{u v}\left(a_{0}^{2}+m a_{0}^{1}\right)\right)\left(\begin{array}{c}
-1 \\
1
\end{array}\right) \cdot\left(\begin{array}{l}
1 \\
p
\end{array}\right) \cos ^{2}(\pi x) d x \\
= & C\left[\left(F_{u u} a_{2}^{1}+F_{u v}\left(a_{2}^{2}+m a_{2}^{1}\right)\right)+2\left(F_{u u} a_{0}^{1}+F_{u v}\left(a_{0}^{2}+m a_{0}^{1}\right)\right)\right](1+p) / 4 \\
K_{2}= & \int 4 A^{2} \kappa \pi^{2}\left(\begin{array}{c}
\alpha_{u} \\
\mu_{c} \alpha_{v}
\end{array}\right) \cdot\left(\begin{array}{l}
1 \\
p
\end{array}\right) x^{2} \cos (\pi x) \cos (\pi x) d x \\
= & -4 C^{2} \kappa\left(\alpha_{u}+\mu_{c} \alpha_{v} p\right)\left[\frac{1}{4}+\frac{\pi^{2}}{6}\right] \\
K_{3}= & \int 2 C^{2} \kappa \pi\left(\begin{array}{c}
\alpha_{u} \\
\mu_{c} \alpha_{v}
\end{array}\right) \cdot\left(\begin{array}{l}
1 \\
p
\end{array}\right) 2 x \sin (\pi x) \cos (\pi x) d x \\
= & C^{2} \kappa\left(\alpha_{u}+\mu_{c} \alpha_{v} p\right) \\
K_{4}= & \int D \tau_{1}^{1} C \pi^{2}\left(\begin{array}{c}
0 \\
m
\end{array}\right) \cdot\left(\begin{array}{l}
1 \\
p
\end{array}\right) \cos ^{2}(\pi x) d x \\
= & C D \pi^{2} p m \tau_{1}^{1} / 2,
\end{aligned}
$$

and $\underline{a}_{2}=\left(a_{2}^{1}, a_{2}^{2}\right)$. Therefore we require that

$$
\tau_{1}^{1}=-2 \frac{\left(K_{1}+K_{2}+K_{3}\right)}{C D m p \pi^{2}} .
$$

Except in special cases, $\tau_{1}^{1} \neq 0$, so that $\varepsilon \eta \tau_{1}^{1}$ is a leading order term in the expansion of $\mu$. Since $\tau_{1}^{0}=0$, the other leading order term will be $\varepsilon^{2} \tau_{2}^{0}$, provided $\tau_{2}^{0} \neq 0$. To determine $\tau_{2}^{0}$, we consider the $O\left(\varepsilon^{3}\right)$ expansion of the model equations. Considering terms of $O\left(\varepsilon^{3} \eta^{0}\right)$, the solvability condition is

$$
\begin{aligned}
& \frac{D m p \pi^{2} \tau_{2}^{0}}{2}+(-1+p) \kappa C^{2}\left(F_{u u}+F_{u v} m\right)\left(\frac{\alpha_{u}}{8}+\frac{\beta_{u}}{4}\right) \\
& \quad+(-1+p) \kappa C^{2} F_{u v}\left(\frac{\alpha_{v}}{8}+\frac{\beta_{v}}{4}\right)+(-1+p) F_{u u v} \frac{3 C^{2}}{16}=0 .
\end{aligned}
$$

Again except in special cases, $\tau_{2}^{0} \neq 0$, and so to leading order $\mu=\mu_{B}^{x}+\varepsilon \eta \tau_{1}^{1}+\varepsilon^{2} \tau_{2}^{0}$. 


\section{A1.2 The branch bifurcating at $\mu_{B}^{y}$}

To approximate the solution near $\mu_{B}^{y}$, we observe, from equations (3.1) and (3.4), that

$$
\left(\begin{array}{l}
u_{1}^{0} \\
v_{1}^{0}
\end{array}\right)=\left(\begin{array}{c}
1 \\
m
\end{array}\right) C \cos (\pi y)
$$

and $\left(u_{1}^{1}, v_{1}^{1}\right)$ is a solution of the system

$$
L\left(\begin{array}{c}
u_{1}^{1} \\
u_{1}^{1}
\end{array}\right)=x^{2}\left(\begin{array}{c}
1 \\
\mu_{c} m
\end{array}\right) \mathrm{C} \pi^{2} \cos (\pi y)+D w_{1}^{y}\left(\begin{array}{c}
0 \\
m
\end{array}\right) \mathrm{C} \pi^{2} \cos (\pi y) .
$$

Here the definition of $w_{1}^{y}$ given in (3.4b) guarantees the existence of a solution to equation (A.6). Again, to determine this solution we express $\left(u_{1}^{1}, v_{1}^{1}\right)$ as the Fourier cosine expansion

$$
\left(\begin{array}{l}
u_{1}^{1} \\
v_{1}^{1}
\end{array}\right)=\sum_{k, j \in \mathbb{N}}\left(\begin{array}{l}
b_{k, j}^{1} \\
b_{k, j}^{2}
\end{array}\right) \cos (k \pi x) \cos (j \pi y)
$$

and substitute this expression into equation (2.1). This gives rise to a series of linear equations for the $\underline{b}_{j, k}$ of the form

$$
\begin{aligned}
M_{k, j}\left(\begin{array}{c}
b_{k, j}^{1} \\
b_{k, j}^{2}
\end{array}\right) \cos (k \pi x) \cos (j \pi y)= & D w_{1}^{y}\left(\begin{array}{c}
0 \\
m
\end{array}\right) C \pi^{2} \cos (\pi y) \\
& +x^{2}\left(\begin{array}{c}
1 \\
\mu_{c} m
\end{array}\right) C \pi^{2} \cos (\pi y),
\end{aligned}
$$

where the matrices $M_{k, j}$ are defined by (A.2). For $(k, j) \neq(0,1)$ and $(k, j) \neq(1,0)$, the matrices $M_{k, j}$ are invertible. In this case, (A.7) may be solved for each $b_{k, j}$ by multiplying each side of the equation by $\cos (k \pi x) \cos (j \pi y)$ and integrating over the unit square. When $j=1$ and $k \neq 0$, we find that $M_{k, j}\left(b_{k, j}^{1}, b_{k, j}^{2}\right)=\left(2 C / k^{2}\right) \cos (k \pi)\left(1, \mu_{c} m\right)$, and when $j \neq 0$ or 1 and $k \neq 0$, we find $\underline{b}_{j, k}=0$. When $(k, j)=(0,1)$ or $(k, j)=(1,0), M_{k, j}$ is singular and we look for a solution of the form

$$
\left(\begin{array}{l}
b_{k, j}^{1} \\
b_{k, j}^{2}
\end{array}\right)=\left(\begin{array}{c}
1 \\
m
\end{array}\right) \mathscr{A}+\left(\begin{array}{c}
1 \\
-1 / m
\end{array}\right) \mathscr{B} .
$$

In the first case, $\mathscr{A}=0$ by orthogonality but in general $\mathscr{B}$ is non-zero and may be found by substitution. When $(k, j)=(1,0)$, it is obvious that $\mathscr{A}=\mathscr{B}=0$. Thus we find

$$
\left(\begin{array}{l}
u_{1}^{1}(\underline{x}) \\
v_{1}^{1}(\underline{x})
\end{array}\right)=\sum_{k} \underline{b}_{k, 1} \cos (k \pi x) \cos (\pi y) .
$$


In particular this implies that the truncated power series approximation (3.9) is dependent on both spatial variables, as discussed in Sect. 3. For $|\eta| \ll 1$, however, the dependence on $x$ is very small, so that the solutions are approximately one-dimensional.

Determining the leading order approximation for $\mu$ in a manner analogous to the above, we find that the solvability condition forces $\tau_{1}^{1}=0$. From consideration of higher order expansions in $\eta$, it is found that $\tau_{1}^{j}=0$ for all $j=2,3, \ldots$. Thus the truncated approximation for $\mu$ reduces to $\mu=\mu_{c}+\varepsilon^{2} \tau_{2}^{0}$.

The value of $\tau_{2}^{0}$ is determined by the $O\left(\varepsilon^{2}\right)$ expansion of the model equations and is given by

$$
\begin{aligned}
& \frac{D m p \pi^{2} \tau_{2}^{0}}{2}+(-1+p) \kappa C^{2}\left(F_{u u}+F_{u v} m\right)\left(\frac{\alpha_{u}}{8}+\frac{\beta_{u}}{4}\right) \\
& \quad+(-1+p) \kappa C^{2} F_{u v}\left(\frac{\alpha_{v}}{8}+\frac{\beta_{v}}{4}\right)+(-1+p) F_{u u v} \frac{3 C^{2}}{16}=0,
\end{aligned}
$$

By comparison with equation (A.5), it is seen that this value of $\tau_{2}^{0}$ is the same as that for the primary solution branch bifurcating from $\mu_{B}^{x}$, and as explained in the main text, we denote this common value by $\tau_{2}^{x}$.

\section{Appendix 2}

Here, we summarise the analysis involved in determining the stability of the various solution branches that we have described for (2.1). The analytical techniques are standard (see, for example, Sattinger, 1972); we consider only linear stability. For the primary branches, linear stability $\underline{u}(\underline{x}, \varepsilon, \eta)$ is determined by considering the eigenvalue problem

$$
\begin{gathered}
\nabla \cdot(\mathscr{D}(x, \eta) \nabla \hat{u})+F_{u}(\underline{u}(\underline{x}, \varepsilon, \eta)) \hat{u}+F_{v}(\underline{u}(\underline{x}, \varepsilon, \eta)) \hat{v}=\lambda \hat{u}, \\
\left.\mu \nabla \cdot(\mathscr{D}(x, \eta) \nabla \hat{v})+G_{u}(\underline{u}, \varepsilon, \eta)\right) \hat{u}+G_{v}(\underline{u} \underline{(x, \varepsilon, \eta)) \hat{v}=\lambda \hat{v},} \\
\hat{u}_{x}=\hat{v}_{x}=0 \text { on } x=0,1, \quad \hat{u}_{y}=\hat{v}_{y}=0 \text { on } y=0,1,
\end{gathered}
$$

and the linear stability of the secondary solution branches $\underline{u}(x, \delta, \eta)$ is determined by considering the eigenvalue problem

$$
\begin{gathered}
\nabla \cdot(\mathscr{D}(x, \eta) \nabla \hat{u})+F_{u}(\underline{\bar{u}}(\underline{x}, \delta, \eta)) \hat{u}+F_{v}(\underline{\bar{u}}(\underline{x}, \delta, \eta)) \hat{v}=\lambda \hat{u}, \\
\left.\mu \nabla \cdot(\mathscr{D}(x, \eta) \nabla \hat{v})+G_{u}(\underline{\bar{u}}, \delta, \eta)\right) \hat{u}+G_{v}(\underline{\bar{u}}(\underline{x}, \delta, \eta)) \hat{v}=\lambda \hat{v}, \\
\hat{u}_{x}=\hat{v}_{x}=0 \text { on } x=0,1, \quad \hat{u}_{y}=\hat{v}_{y}=0 \text { on } y=0,1 .
\end{gathered}
$$


In both cases $\lambda$ represents the rate of linear growth of perturbations $\hat{u}(x)$ about the steady state solutions. In particular, the solution branch is stable if and only if $\lambda<0$ for all perturbation solutions.

To solve the eigenvalue problem for $\lambda$, we substitute the power series expansions for the primary and secondary solution branches into equation (B.9) or (B.10) as appropriate, and assume that both $\lambda$ and $\hat{u}(x)$ may be expressed as power series expansions in the same small parameters. Thus on the primary solution branches, we look for solutions of the eigenvalue problem of the form

$$
\begin{aligned}
\underline{\hat{u}}(\underline{x}, \varepsilon, \eta) & =\varepsilon \underline{\hat{u}}_{1}(\underline{x}, \eta)+\varepsilon^{2} \hat{\hat{u}}_{2}(\underline{x}, \eta)+O\left(\varepsilon^{3}\right) \\
\lambda(\varepsilon, \eta) & =\lambda_{0}(\eta)+\varepsilon \lambda_{1}(\eta)+\varepsilon^{2} \lambda_{2}(\eta)+O\left(\varepsilon^{3}\right),
\end{aligned}
$$

satisfying $\left\|\underline{\hat{u}}_{1}\right\|=1$, where for $i=1,2, \ldots$ and $j=0,1,2, \ldots$

$$
\begin{aligned}
\hat{u}_{i}(\underline{x}, \eta) & =\underline{\hat{u}}_{i}^{0}+\eta \hat{u}_{i}^{1}+\eta^{2} \hat{u}_{i}^{2}+O\left(\eta^{3}\right) \\
\lambda_{j}(\eta) & =\lambda_{j}^{0}+\eta \lambda_{j}^{1}+\eta^{2} \lambda_{j}^{2}+O\left(\eta^{3}\right) .
\end{aligned}
$$

On the secondary solution branches, the appropriate power series expansions are

$$
\begin{aligned}
\underline{\hat{u}}(\underline{x}, \delta, \eta) & =\delta \underline{\hat{u}}_{1}(\underline{x}, \eta)+\delta^{2} \underline{\hat{u}}_{2}(\underline{x}, \eta)+O\left(\delta^{3}\right) \\
\lambda(\delta, \eta) & =\lambda_{0}(\eta)+\delta \lambda_{1}(\eta)+\delta^{2} \lambda_{2}(\eta)+O\left(\varepsilon^{3}\right),
\end{aligned}
$$

satisfying $\left\|\hat{u}_{1}\right\|=1$, where for $i=1,2, \ldots$ and $j=0,1,2, \ldots$

$$
\begin{aligned}
& \hat{\hat{u}}_{i}(\eta)=\eta^{1 / 2} \hat{u}_{i}^{1}+\eta \hat{\hat{u}}_{i}^{2}+O\left(\eta^{3 / 2}\right) \\
& \lambda_{j}(\eta)=\eta^{1 / 2} \lambda_{j}^{1}+\eta \lambda_{j}^{2}+O\left(\eta^{3 / 2}\right) .
\end{aligned}
$$

Substituting these expressions into the eigenvalue equations, the twovariable perturbation technique may then be used to determine the leading order approximation for $\lambda$. Specifically, we approximate $\lambda$ on the primary solution branches by

$$
\lambda=\eta \lambda_{0}^{1}+\varepsilon \eta \lambda_{1}^{1}+\varepsilon^{2} \lambda_{2}^{0},
$$

and on the secondary solution branches by

$$
\lambda=\eta \lambda_{0}^{2}+\delta^{2} \eta \lambda_{2}^{2} .
$$

From these approximations we are able to derive the conditions for the stability of the primary and secondary solution branches when $\eta \neq 0$ and compare these with the conditions for the stability of primary striped and spotted solutions when $\eta=0$ (see Sect. 5).

As this analysis is standard, we summarise the results for our system. Considering first the primary solution branch bifurcating from $\mu_{B}^{x}$, we find that its stability for a given value of $\mu$, or equivalently for 
a given value of the parameter $\varepsilon$, is determined by the signs of $\lambda_{x}$ and $\lambda_{y}$, where

$$
\begin{aligned}
& \lambda_{x}=\frac{D \pi^{2} m p}{1+m p}\left\{\varepsilon \eta \tau_{1}^{1}+\varepsilon^{2} 2 \tau_{2}^{x}\right\} \\
& \lambda_{y}=\frac{D \pi^{2} m p}{1+m p}\left\{-\eta\left(w_{1}^{x}-w_{1}^{y}\right)+2 \varepsilon^{2}\left(\tau_{2}^{x y}-\tau_{2}^{x}\right)\right\} .
\end{aligned}
$$

When both $\lambda_{x}<0$ and $\lambda_{y}<0$ the spatially inhomogeneous steady state solution is stable; otherwise it is unstable. Equation (B.13) implies that

$$
\lambda_{x}<0 \Leftrightarrow \tau_{2}^{x}>0 \text { and } \varepsilon \notin\left[\varepsilon_{L}, 0\right],
$$

where $\varepsilon_{L}=-\eta \tau_{1}^{1} /\left(2 \tau_{2}^{x}\right)$ is the position of the limit point denoted by $\mu=\mu_{L}$; for $\tau_{2}^{x}>0$, the parameter interval $\left[\varepsilon_{L}, 0\right]$ corresponds to the lower part of the subcritical region of the primary solution branch (see Fig. 1). Here we have used the fact that $m p \in(-1,0)$ for all reaction kinetics which exhibit diffusion driven instability; this follows from simple algebraic manipulation of the equation (3.2) defining $m$ and the corresponding expression for $p$ (given immediately before (3.4)). Similarly, we can deduce that

$$
\begin{aligned}
& \lambda_{y}<0 \Leftrightarrow \begin{array}{c}
\left(\tau_{2}^{x y}-\tau_{2}^{x}\right)<0 \text { and } \varepsilon^{2}<\varepsilon_{x}^{2}, \\
\left(\tau_{2}^{x y}-\tau_{2}^{x}\right)>0 \text { and } \varepsilon^{2}>\varepsilon_{x}^{2}
\end{array}, \quad \text { if } \varepsilon_{x}^{2}>0 \\
& \lambda_{y}<0 \Leftrightarrow\left(\tau_{2}^{x y}-\tau_{2}^{x}\right)>0 \forall \varepsilon \text { if } \varepsilon_{x}^{2}<0,
\end{aligned}
$$

where

$$
\varepsilon_{x}=\left[\frac{\eta\left(w_{1}^{x}-w_{1}^{y}\right)}{2\left(\tau_{2}^{x y}-\tau_{2}^{x}\right)}\right]^{1 / 2}
$$

defines the position of the secondary bifurcation point $\mu_{S}^{x}$ when

$$
\frac{w_{1}^{x}-w_{1}^{y}}{\tau_{2}^{x y}-\tau_{2}^{x}}>0
$$

Thus, when $\mu_{S}^{x}$ exists, the primary solution branch is stable for $\mu_{L}<\mu<\mu_{S}^{x}$ if and only if $\tau_{2}^{x}>0, \tau_{2}^{x}>\tau_{2}^{x y}$, and for $\mu>\mu_{S}^{x}$ if and only if $\tau_{2}^{x y}>\tau_{2}^{x}>0$. When $\mu_{S}^{x}$ does not exist, the solution branch is stable for all $\mu>\mu_{B}^{x}$ whenever $\tau_{2}^{x y}>\tau_{2}^{x}>0$. In particular, it follows that a necessary condition for stability is $\tau_{2}^{x}>0$.

By a similar analysis, we may determine the stability conditions for the primary solution branch bifurcating from $\mu_{B}^{v}$; the results are outlined in Sect. 5.

Using the above ideas we can also analyse the stability of the solutions on the secondary branches. First we determine the stability of the secondary solution branch bifurcating from $\mu_{S}^{x}$, which has power 
series expansion (2.6). We find that the stability of the secondary solution branch is determined by the signs of $\lambda_{x}$ and $\lambda_{y}$, where

$$
\begin{aligned}
\lambda_{x}(\delta, \eta) & =\eta \frac{2 D \pi^{2} m p \tau_{2}^{x}}{1+m p}-\delta^{2} \eta \frac{8 D m p \pi^{2} \tau_{2}^{x y}\left(\tau_{2}^{x y}-\tau_{2}^{x}\right)}{(1+m p) \tau_{2}^{x}} \\
\lambda_{y}(\delta \eta) & =-\delta^{2} \eta \frac{8 D m p \pi^{2} \tau_{2}^{x y}\left(\tau_{2}^{x y}-\tau_{2}^{x}\right)}{(1+m p) \tau_{2}^{x}} .
\end{aligned}
$$

The solutions are stable if both $\lambda_{x}<0$ and $\lambda_{y}<0$, and are unstable otherwise. It follows that for sufficiently small $\delta, \lambda_{x}<0 \Leftrightarrow \tau_{2}^{x}>0$. As before, we have used the fact that, for all parameter values lying in the generalised Turing space, $m p \in(-1,0)$. Similarly, $\lambda_{y}<0 \Leftrightarrow$ $\tau_{2}^{x y}\left(\tau_{2}^{x y}-\tau_{2}^{x}\right) / \tau_{2}^{x}<0$. Thus close to the secondary bifurcation point, where $\delta \ll 1$, there exist stable spotted solutions for $\mu>\mu_{S}^{x}$ if and only if $\tau_{2}^{x}>\tau_{2}^{x y}>0$.

Acknowledgements. DLB was supported in part by a Wellcome Trust Prize Studentship. Part of this work was carried out while PKM was visiting the Department of Mathematics, Williams College, Massachusettes. This work was supported in part by a grant from the London Mathematical Society.

\section{References}

Bauer, L., Keller, H. B. and Reiss, E. L., 1975, Multiple eigenvalues lead to secondary bifurcation. SIAM Review, 17, 101-122

Benson, D. L., Sherratt, J. A. and Maini, P. K., 1993, Diffusion driven instability in an inhomogeneous domain. Bull. Math. Biol., 55, 365-384

Boissonade, J., 1994, Long-range inhibition. Nature, 369, 188-189

Borckmans, P., De Wit, A. and Dewel, G., 1992, Competition in ramped Turing structures. Physica A, 188, 137-157

De Wit, A., Dewel, G., Borckmans, P. and Walgraef, D., 1992, 3-Dimensional dissipative structures in reaction diffusion-systems. Physica D, 61, 289-296

Doedel, E., 1976, AUTO: software for continuation and bifurcation problems in ordinary differential equations. Technical report, California Institute of Technology

Dufiet, V., and Boissonade, J., 1992a, Conventional and unconventional Turing patterns. J. Chem. Phys., 96, 664-673

Dufiet, V. and Boissonade, J., 1992b, Numerical studies of Turing pattern selection in a two-dimensional system. Physica A, 118, 158-171

Ermentrout, B., 1991, Stripes or spots? Nonlinear effects of bifurcation of reaction diffusion equations on the square. Proc. R. Soc. Lond., A434, 413-417

Gierer, A. and Meinhardt, H., 1972, A theory of biological pattern formation. Kybernetik, 12, 30-39

Hunding, A. and Brøns, M., 1990, Bifurcation in a spherical reaction diffusion system with imposed gradient. Physica D, 44, 285-302

Iooss, G. and Joseph, D. D., 1980, Elementary stability and bifurcation theory, New York: Springer-Verlag 
Jenson, O., Pannbacker, V. O., Dewel, G. and Borckmans, P., 1993, Subcritical transitions to Turing structures. Phys. Lett. A, 179, 91-96

Lyons, M. J. and Harrison, L. G., 1991, A class of reaction diffusion models which preferentially select striped patterns. Chem. Phys. Lett., 183, 159-164

Lyons, M. J. and Harrison, L. G., 1992, Stripe selection: An intrinsic property of some pattern-forming models with non-linear dynamics. Devel. Dyn., 195, 201-215

Mahar, T. J. and Matkowsky, B. J., 1977, A model biochemical reaction exhibiting secondary bifurcation. SIAM Review, 32, 394-404

Malomed, B. A. and Tribel'skii, M. I., 1987, Stability of stationary periodic structures for weakly supercritical convection and in related problems. Sov. Phys. JETP, 65, 305-310

Maini, P. K., Benson, D. L. and Sherratt, J. A., 1992, Pattern formation in reactiondiffusion models with spatially inhomogeneous diffusion coefficients. IMA J. Math. Appl. Med. Biol., 9, 197-213

Maini, P. K., Myerscough, M. R., Winters, K. H. and Murray, J. D., 1991, Bifurcating spatially heterogeneous solutions in a chemotaxis model for biological pattern generation. Bull. Math. Biol., 53, 701-719

Meinhardt, H., 1993, A model for pattern formation of hypostome, tentacles, and foot in hydra - how to form structures close to each other, how to form them at a distance. Dev. Biol., 157, 321-333

Murray, J. D., 1981, A pre-pattern formation mechanism for animal coat markings. J. theor. Biol., 88, 161-199

Murray, J. D., 1982, Parameter space for Turing instability in reaction diffusion mechanisms: A comparison of models. J. theor. Biol., 98, 143-163

Murray, J. D., 1989, Mathematical Biology. Heidelberg: Springer-Verlag

Nagorcka, B. N., 1995a, The reaction-diffusion theory of wool (hair) follicle initiation and development. 1. primary follicles. Austr. J. Agric. Res., 46, 333-355

Nagorcka, B. N., 1995b, The reaction-diffusion theory of wool (hair) follicle initiation and development. 2. original secondary follicles. Austr. J. Agric. Res., 46, 357-378

Perraud, J. J., De Wit, A., De Kepper, P., Dewel G. and Borckmans, P., 1993, Onedimensional spirals; Novel asynchronous chemical waves sources. Phys. Rev. Lett., 71, 1272-1275

Reiss, E. L., 1983, Cascading bifurcations. SIAM J. Appl. Math., 43, 57-65

Sattinger, D. H., 1972, Six lectures on the transition to instability. In Lecture Notes in Mathematics, 322, Berlin: Springer-Verlag

Schnakenberg, J., 1979, Simple chemical reaction systems with limit cycle behaviour. J. Theor. Biol., 81, 389-400

Segel, L. A. and Jackson, J. L., 1972, Dissipative structure: an explanation and an ecological example. J. Theor. Biol., 37, 545-559

Sherratt, J. A., 1995, Turing bifurcations with a temporally varying diffusion coefficient. J. Math. Biol., 33, 295-308

Turing, A. M., 1952, The chemical basis of morphogenesis. Phil. Trans. R. Soc. Lond., B237, 37-72 